\title{
Neutral Higgs production at proton colliders in the CP-conserving NMSSM
}

\author{
Stefan Liebler ${ }^{\mathrm{a}}$ \\ DESY, Notkestraße 85, 22607 Hamburg, Germany
}

Received: 11 March 2015 / Accepted: 26 April 2015 / Published online: 14 May 2015

(C) The Author(s) 2015. This article is published with open access at Springerlink.com

\begin{abstract}
We discuss neutral Higgs boson production through gluon fusion and bottom-quark annihilation in the CP-conserving $\mathbb{Z}_{3}$-invariant Next-to-Minimal Supersymmetric Standard Model at proton colliders. For gluon fusion we adapt well-known asymptotic expansions in supersymmetric particles for the inclusion of next-to-leading order contributions of squarks and gluinos from the Minimal Supersymmetric Standard Model (MSSM) and include electroweak corrections involving light quarks. Together with the resummation of higher-order sbottom contributions in the bottom-quark Yukawa coupling for both production processes we thus present accurate cross section predictions implemented in a new release of the code SusHi. We elaborate on the new features of an additional $\mathrm{SU}(2)_{L}$ singlet in the production of CP-even and -odd Higgs bosons with respect to the MSSM and include a short discussion of theoretical uncertainties.
\end{abstract}

\section{Introduction}

After the discovery of a scalar boson at the Large Hadron Collider (LHC) [1,2] in 2012 an essential task of particle physicists is to reveal the nature of the Higgs-like state and thus the nature of electroweak symmetry breaking. Apart from deviations from the Standard Model (SM) prediction of the properties of the found Higgs-like state, further work includes the search for additional less and/or more massive scalar bosons, which can nicely be accommodated in supersymmetric models. The Next-to-Minimal Supersymmetric Standard Model (NMSSM) extends the Minimal Supersymmetric Standard Model (MSSM) by an $\mathrm{SU}(2)_{L}$ singlet and allows the dynamical generation of the $\mu$-term through electroweak symmetry breaking [3,4]. The latter singlet-doublet mixing term in the superpotential lifts the MSSM tree-level upper bound of the Higgs mass given by the $Z$-boson mass.

a e-mail: stefan.liebler@desy.de
Thus, the NMSSM can easily accommodate the SM-like Higgs boson with a mass close to $125 \mathrm{GeV}$. Whereas for the calculation of the NMSSM Higgs spectrum and branching ratios various spectrum generators are available, including higher orders in perturbation theory (see Sect. 3), the calculation of neutral Higgs production cross sections did not exceed the leading order (LO) in quantum chromodynamics involving squarks and gluinos (SQCD) [5] and did not include electroweak corrections-apart from private implementations in e.g. HIGLU [6].

It is therefore timely to present the missing ingredients and a code for the calculation of accurate neutral Higgs production cross sections in the NMSSM, where the five neutral Higgs bosons are predominantly generated through gluon fusion and bottom-quark annihilation at a proton collider. For this purpose we extend the code SusHi [7]. For the time being we restrict our implementation to the real NMSSM without additional $\mathrm{CP}$ violation, such that $\mathrm{CP}$-even $H_{1}, H_{2}$, and $H_{3}$ and CP-odd Higgs bosons $A_{1}$ and $A_{2}$ can be distinguished in the Higgs sector. Most recent efforts related to Higgs physics at the LHC are summarized in the reports of the LHC Higgs cross section working group [8-10]. The SM Higgs is mainly produced through gluon fusion, where the Higgs-gluon coupling is mediated through virtual top and bottom quarks [11]. Higher-order QCD corrections at nextto-leading order (NLO) are of large importance [12-14]. In the effective theory of a heavy top quark the inclusive cross section is known to next-to-next-to-leading order (NNLO) in QCD [15-17], in addition finite top-quark mass effects at NNLO were calculated [18-22]. Beyond NNLO QCD effects are accessible through resummation [23-28] and electroweak corrections are known [29-31]. Meanwhile next-to-NNLO (NNNLO) QCD contributions were estimated in the so-called threshold expansion [32-35], but they are not further considered in this publication.

The SM results for Higgs production through gluon fusion can be adjusted to the MSSM and the NMSSM through a 
proper reweighting of the Higgs couplings to the quarks. However, the gluon fusion process can also be mediated through their superpartners, the squarks. With respect to the MSSM the only generically new ingredient, which goes beyond the projection of the physical Higgs bosons onto the neutral components of the two Higgs doublets, are couplings of the NMSSM singlet to squarks, since no couplings of the singlet to quarks or gauge bosons are present in the treelevel Lagrangian. It is therefore of importance to include squark contributions to gluon fusion at the highest order possible, even though they decrease in size with increasing squark masses. For the pseudoscalars $A_{i}$ squark contributions to gluon fusion are only induced at NLO, which motivates to go beyond just LO squark contributions for all Higgs bosons. For this purpose we adapt the works of Refs. [3638] for the MSSM to present NLO SQCD contributions for the NMSSM, which are based on an expansion in terms of heavy supersymmetric particles taking into account terms up to $\mathcal{O}\left(m_{\phi}^{2} / M^{2}\right), \mathcal{O}\left(m_{t}^{2} / M^{2}\right), \mathcal{O}\left(m_{b}^{2} / M^{2}\right)$ and $\mathcal{O}\left(m_{Z}^{2} / M^{2}\right)$, where $m_{\phi}$ denotes the Higgs mass and $M$ a generic SUSY mass. In contrast to the MSSM we are at present only working in this expansion of inverse SUSY masses and do not include an expansion in the so-called VHML, the vanishing Higgs mass limit $\left(m_{\phi} \rightarrow 0\right)$ for the SQCD contributions, as implemented in evalcsusy [39-41] or discussed in Ref. [42]. In the latter limit higher-order stop-induced contributions up to NNLO level are known [43-45] and were partially included in previous discussions of precise MSSM neutral Higgs production cross sections [46]. Although for a pure CP-odd singlet component NNLO stop-induced contributions are the first non-vanishing contributions to the gluon fusion cross section, we leave an inclusion of these to future work. For completeness, we add that in the MSSM a numerical evaluation of NLO squark/quark/gluino contributions was also reported in Refs. [47,48], whereas Refs. [49-51] presented analytic results for the pure squark-induced NLO contributions. Electroweak contributions to the gluon-fusion production process mediated through light quarks $[30,31]$ can be adjusted from the SM to the MSSM [7] and similarly to the NMSSM and are known to capture the dominant fraction of electroweak contributions for a light SM-like Higgs with a mass below the top-quark mass, whereas they are generically small for larger Higgs masses.

For large values of $\tan \beta$, the ratio of the vacuum expectation values of the neutral components of the two Higgs doublets, the bottom-quark Yukawa coupling is enhanced, such that the bottom sector gets more important for gluon fusion, and the associated production with a pair of bottom quarks $p p \rightarrow b \bar{b} \phi$ is significantly enhanced. SusHi includes bottom-quark annihilation $b \bar{b} \rightarrow \phi$, which in the case of non-tagged final state $b$-quarks is a good theoretical approach, since it resums logarithms through the $b$-parton distribution functions. The latter process is known as five- flavor scheme (5FS) up to NNLO QCD [52,53] and can easily be reweighted from the SM to the MSSM/NMSSM by effective couplings $[54,55]$. In the NMSSM the singlet does not couple to the quarks at LO, however, taking into account the singlet-induced component into the resummation of higherorder sbottom effects is mandatory, since also the singlet to sbottom couplings are enhanced by $\tan \beta$.

The new release of SusHi thus provides gluon-fusion cross sections at NLO QCD taking into account the third generation quarks and their superpartners, the squarks, for all the five neutral Higgs bosons of the NMSSM. The squark and squark/quark/gluino contributions are implemented in asymptotic expansions of heavy SUSY masses. Electroweak corrections induced by light quarks through the couplings of the Higgs bosons to $Z$ and $W^{ \pm}$bosons can be added consistently like in the MSSM. Similarly, the NNLO top-quark induced contributions are included. In addition, sbottom contributions can be resummed into an effective bottom-quark Yukawa coupling, also taking into account the additional singlet to sbottom couplings. The latter also applies to the calculated bottom-quark annihilation cross section at NNLO QCD. All features SusHi provides for the MSSM are available for the NMSSM as well, in particular distributions with respect to the (pseudo)rapidity and transverse momentum of the Higgs boson under consideration can be obtained. Left for future work is a link to MoRe-SusHi [56] to allow for the calculation of momentum resummed transverse momentum distributions.

We proceed as follows: We start with a discussion of the theory background in Sect. 2, where we elaborate on the NMSSM Higgs sector and the calculation of the gluon-fusion cross section. Then we present the NLO virtual amplitude for gluon fusion as well as the calculation of bottom-quark annihilation including the resummation of sbottom-induced contributions to the bottom-quark Yukawa coupling in the NMSSM. Subsequently we comment on the implementation in SusHi in Sect. 3, before we investigate the phenomenological features of the singlet-like Higgs boson in the CPeven and CP-odd sector with regard to Higgs production in Sect. 4. We also include a short discussion of theoretical uncertainties. Finally, we conclude and present the Higgssquark-squark couplings in Appendix A.

\section{Theory background}

In this section we discuss the Higgs sector of the CPconserving $\mathbb{Z}_{3}$-invariant NMSSM, before we proceed to the resummation of $\tan \beta$ enhanced sbottom contributions in the bottom-quark Yukawa coupling. Subsequently we move to the discussion of the Higgs production cross section in gluon fusion, where we present the adapted formulas for the NLO SQCD virtual amplitude, and finally comment on 
the consequences of the additional singlet to bottom-quark annihilation.

\subsection{The Higgs sector of the CP-conserving NMSSM}

Our notation of the Higgs sector of the CP-conserving $\mathbb{Z}_{3^{-}}$ invariant NMSSM closely follows Ref. [57]. For NMSSM reviews we refer to Refs. $[3,4]$. The superpotential can be written in the form

$W_{\mathrm{NMSSM}}=W_{\mathrm{MSSM}}-\epsilon_{a b} \lambda \hat{S} \hat{H}_{d}^{a} \hat{H}_{u}^{b}+\frac{1}{3} \kappa \hat{S}^{3}$,

where $W_{\text {MSSM }}$ equals the superpotential of the MSSM without $\mu$-term. $\hat{S}$ denotes the additional $\mathrm{SU}(2)_{L}$ singlet superfield compared to the MSSM with the two $\mathrm{SU}(2)_{L}$ doublet superfields $\hat{H}_{d}$ and $\hat{H}_{u} . \epsilon_{a b}$ contracts the $\mathrm{SU}(2)_{L}$ doublet components. Since the singlet $\hat{S}$ is a neutral field, it induces one additional $\mathrm{CP}$-even and one additional $\mathrm{CP}$-odd neutral Higgs boson as well as one additional neutralino compared to the MSSM. The soft-breaking terms include the scalar components $H_{d}, H_{u}$, and $S$ of the superfields and are given by

$$
\begin{aligned}
\mathcal{L}_{\text {soft }}= & \mathcal{L}_{\text {soft, MSSM }}+\left(\epsilon_{a b} \lambda A_{\lambda} S H_{d}^{a} H_{u}^{b}-\frac{1}{3} \kappa A_{\kappa} S^{3}+\text { h.c. }\right) \\
& -m_{s}^{2}|S|^{2} .
\end{aligned}
$$

The soft-breaking mass $m_{s}$ can be derived from the minimization conditions of the tadpole equations (in addition to $m_{H_{d}}^{2}$ and $m_{H_{u}}^{2}$ like in the MSSM), whereas $A_{\lambda}$ and $A_{\kappa}$ are usually considered input parameters. $A_{\lambda}$ can be alternatively replaced by the charged Higgs mass $m_{H^{ \pm}}$as input parameter. The neutral components of the Higgs fields are decomposed according to

$H_{d}^{0}=\frac{1}{\sqrt{2}}\left(v_{d}+H_{d}^{R}+i H_{d}^{I}\right)$

$H_{u}^{0}=\frac{1}{\sqrt{2}}\left(v_{u}+H_{u}^{R}+i H_{u}^{I}\right)$,

$S=\frac{1}{\sqrt{2}}\left(v_{s}+S^{R}+i S^{I}\right)$,

where $v_{d}, v_{u}$, and $v_{s}$ denote the vacuum expectation values (VEVs) and the fields with indices $R$ and $I$ are the CP-even and CP-odd fluctuations around them. An effective $\mu$ term is generated through the VEV of the singlet

$\mu=\frac{1}{\sqrt{2}} \lambda v_{s}$,

which will be further used within this article. We do not present the explicit form of the mass matrices here, but refer to Ref. [57]. We define the CP-even gauge eigenstate basis $H^{R}=\left(H_{d}^{R}, H_{u}^{R}, S^{R}\right)$ and the CP-odd one $H^{I}=$
$\left(H_{d}^{I}, H_{u}^{I}, S^{I}\right)$. Whereas in the former case the mass eigenstates $H_{i}$ with $i \in\{1,2,3\}$ are obtained through one rotation

$H_{i}=\sum_{j=1}^{3} \mathcal{R}_{i j}^{S} H_{j}^{R}$

we perform a prerotation in the CP-odd sector to obtain the MSSM pseudoscalar $A$ and the Goldstone $G$ in the form $H^{\prime I}=\left(G, A, S^{I}\right)$. The prerotation $\mathcal{R}^{G}$ is given by the ratio of vacuum expectation values $\tan \beta=v_{u} / v_{d}$, such that the mass eigenstates $A_{i}$ with $i \in\{1,2\}$ are obtained by

$$
\begin{gathered}
A_{i}=\sum_{j=1}^{3} \mathcal{R}_{i+1, j}^{P} H_{j}^{\prime I}=\sum_{j, k=1}^{3} \mathcal{R}_{i+1, j}^{P} \mathcal{R}_{j k}^{G} H_{k}^{I} \\
\text { with } \mathcal{R}^{G}=\left(\begin{array}{ccc}
c_{\beta} & -s_{\beta} & 0 \\
s_{\beta} & c_{\beta} & 0 \\
0 & 0 & 1
\end{array}\right),
\end{gathered}
$$

where $\mathcal{R}^{P}$ is a $(3 \times 3)$-matrix, which, however, only consists of a $(2 \times 2)$-mixing block, whereas $\mathcal{R}_{i 1}^{P}=\mathcal{R}_{1 i}^{P}=0$ for $i \neq 1$ and $\mathcal{R}_{11}^{P}=1$. For Higgs production the Goldstone boson does not need to be considered. In the following we make use of the notation "singlet-like Higgs boson", which refers to the $\mathrm{CP}$-even/odd Higgs boson with the dominant fraction of the singlet component $S$ in gauge eigenstates. For this purpose we define the singlet character $\left|\mathcal{R}_{i, 3}^{S}\right|^{2}$ for $H_{i}$ and $\left|\mathcal{R}_{i+1,3}^{P}\right|^{2}$ for $A_{i}$. In our discussion of cross sections we denote the Higgs boson by the letter $\phi$, which can be replaced by any of the physical Higgs bosons $H_{i}$ or $A_{i}$. For picking viable scenarios for phenomenological studies we refer to Ref. [58] for a recipe to obtain positive eigenvalues for the singlet-like CP-even and -odd Higgs by varying $A_{\kappa}$ between a minimal and a maximal value.

Whereas the singlet component $S$ does not couple to quarks, $F$-terms induce a coupling of the singlet-like Higgs to squarks, which is of relevance for Higgs production. We present the Higgs-squark-squark couplings to the third generation of squarks in Appendix A. We point out that the singlet component mixes with the Higgs doublets proportional to $\lambda$ and also the couplings of the singlet component to squarks are proportional to $\lambda$. It is thus possible to mostly decouple the singlet component by lowering the value of the parameter $\lambda$. The couplings to quarks can easily be translated from the MSSM by the correct projection on the neutral doublet components $H_{d}^{R}, H_{u}^{R}$, and the pseudoscalar $A$ and yield relative to the SM

$$
\begin{aligned}
& g_{d}^{H_{i}}=\mathcal{R}_{i 1}^{S} \frac{1}{\cos \beta}, \quad g_{u}^{H_{i}}=\mathcal{R}_{i 2}^{S} \frac{1}{\sin \beta}, \\
& g_{d}^{A_{i}}=\mathcal{R}_{i+1,2}^{P} \tan \beta, \quad g_{u}^{A_{i}}=\mathcal{R}_{i+1,2}^{P} \frac{1}{\tan \beta},
\end{aligned}
$$


with $i \in\{1,2,3\}$ in the CP-even and $i \in\{1,2\}$ in the CPodd Higgs sector. The relative strength $g_{f}^{\phi}$ enters the Yukawa couplings in the form $Y_{f}^{\phi}=\sqrt{2} m_{f} g_{f}^{\phi} / v$ with the vacuum expectation value $v^{2}=v_{d}^{2}+v_{u}^{2}$.

\subsection{Resummation of higher-order sbottom contributions}

It is well known in the MSSM that $\tan \beta$ enhanced sbottom corrections to the bottom-quark Yukawa coupling can be treated in an effective Lagrangian approach [59-64] to be resummed. For the case of the NMSSM just taking into account SQCD corrections the effective Lagrangian can be written in the form [65]

$$
\begin{gathered}
\mathcal{L}_{\text {eff }}=-Y_{b} \bar{b}_{R}\left[H_{d}^{0}+\frac{\lambda \Delta_{b}}{\mu \tan \beta} S^{*} H_{u}^{0 *}\right] b_{L} \quad \text { with } \\
\Delta_{b}=\frac{2}{3} \frac{\alpha_{s}}{\pi} m_{\tilde{g}} \mu \tan \beta I\left(m_{\tilde{b}_{1}}^{2}, m_{\tilde{b}_{2}}^{2}, m_{\tilde{g}}^{2}\right) \quad \text { and } \\
I(a, b, c)=\frac{a b \log \frac{a}{b}+b c \log \frac{b}{c}+c a \log \frac{c}{a}}{(a-b)(b-c)(a-c)} .
\end{gathered}
$$

Reference [65] additionally presents the inclusion of SUSY electroweak corrections proportional to the soft-breaking parameter $A_{t}$. The inclusion of electroweak corrections into $\Delta_{b}$ does not harm our subsequent discussion of SQCD corrections and can thus always be included in the bottom-quark Yukawa coupling entering gluon fusion and bottom-quark annihilation. Apart from a coupling of the bottom quarks to the gauge eigenstate $H_{u}^{0}$ also an effective coupling to the singlet $S$ can be induced at loop level, the latter being proportional to $\lambda v_{u}$ instead of $\mu$. The sbottom corrections can be absorbed into effective Yukawa couplings, which read [65]

$\tilde{g}_{b}^{H_{i}}=\frac{g_{b}^{H_{i}}}{1+\Delta_{b}}\left[1+\Delta_{b}\left(\frac{\mathcal{R}_{i 2}^{S}}{\mathcal{R}_{i 1}^{S} \tan \beta}+\frac{\mathcal{R}_{i 3}^{S} v \cos \beta}{\mathcal{R}_{i 1}^{S} v_{s}}\right)\right]$

for the three CP-even Higgs field $H_{i}$ and

$\tilde{g}_{b}^{A_{i}}=\frac{g_{b}^{A_{i}}}{1+\Delta_{b}}\left[1+\Delta_{b}\left(-\frac{1}{\tan ^{2} \beta}-\frac{\mathcal{R}_{i+1,3}^{P} v}{\mathcal{R}_{i+1,2}^{P} v_{s} \tan \beta}\right)\right]$

for the two CP-odd Higgs fields $A_{i}$.

\subsection{Gluon-fusion cross section}

After our discussion of the CP-conserving NMSSM Higgs sector and the resummation of sbottom contributions in the bottom-quark Yukawa coupling, we present the gluon-fusion production cross section for a Higgs boson $\phi$, which can be written in the form [7]

$$
\begin{aligned}
& \sigma(p p \rightarrow \phi+X)=\sigma_{0}^{\phi}\left[1+C^{\phi} \frac{\alpha_{s}}{\pi}\right] \tau_{\phi} \frac{\mathrm{d} \mathcal{L}^{g g}}{\mathrm{~d} \tau_{\phi}} \\
& +\Delta \sigma_{g g}^{\phi}+\Delta \sigma_{g q}^{\phi}+\Delta \sigma_{q \bar{q}}^{\phi}
\end{aligned}
$$

with $\tau_{\phi}=m_{\phi}^{2} / s$ and the hadronic center-of-mass energy $s$. The factor $\sigma_{0}^{\phi}$ includes the LO partonic cross section. $C^{\phi}$ encodes NLO terms of a singular nature in the limit $\hat{s} \rightarrow m_{\phi}^{2}$ with the partonic center-of-mass energy $\hat{s}$. The gluon-gluon luminosity is given by the integral

$\frac{\mathrm{d} \mathcal{L}^{g g}}{\mathrm{~d} \tau}=\int_{\tau}^{1} \frac{\mathrm{d} x}{x} g(x) g(\tau / x)$.

The contributions $\Delta \sigma_{g g}^{\phi}, \Delta \sigma_{g q}^{\phi}$, and $\Delta \sigma_{q \bar{q}}^{\phi}$ are the regular terms in the limit $\hat{s} \rightarrow m_{\phi}^{2}$ in the partonic cross section and arise from $g g, g q$, and $q \bar{q}$ scattering, respectively. The LO contribution $\sigma_{0}^{\phi}$ is obtained by the formulas presented in Ref. [7] using the NMSSM couplings of the Higgs bosons to the quarks and squarks. Similarly the contributions $\Delta \sigma_{x y}^{\phi}$ are obtained from the MSSM by a proper replacement of the involved Higgs boson to quark and squark couplings. The factor $C^{\phi}$ can be decomposed in the form

$C^{\phi}=2 \operatorname{Re}\left[\frac{\Phi^{(2 l)}}{\Phi_{\infty}^{(1 l)}}\right]+\pi^{2}+\beta_{0} \log \left(\frac{\mu_{R}^{2}}{\mu_{F}^{2}}\right)$,

with $\beta_{0}=11 / 2-n_{f} / 3$ and $n_{f}=5$ as well as the factorization and renormalization scales, $\mu_{F}$ and $\mu_{R}$, respectively. $\Phi_{\infty}^{(1 l)}$ is the LO (one-loop) virtual amplitude in the limit of large stop and sbottom masses. $\Phi^{(2 l)}$ is the NLO (twoloop) virtual amplitude and equals the form factors $\mathcal{H}_{i}^{(2 l)}$ for the CP-even Higgs bosons and $\mathcal{A}_{i}^{(2 l)}$ for the CP-odd Higgs bosons, which are presented in Sect. 2.4 to account for NLO virtual contributions from quarks and squarks in an appropriate way. In the MSSM limit they correspond to the form factors of Refs. [36-38] except from a constant factor of $-3 / 4$. Contrary to the case of the MSSM we do not employ evalcsusy $[40,41]$ to obtain the NLO amplitude in the limit of heavy top-quark and stop masses for the light Higgs, but use the expanded form factors presented in the following sections instead. Accordingly our implementation does not (yet) include approximate NNLO stop contributions as presented in Ref. [46] for the MSSM. The NNLO top-quark contributions in the heavy top-quark effective theory making use of Refs. [15,66] are included according to Eq. (29) of Ref. [7].

Lastly we comment on the inclusion of the electroweak corrections to the gluon-fusion production cross section. Similarly to the MSSM the full SM NLO electroweak (EW) corrections [29] can be added to the top-quark induced result only, assuming complete factorization of $\mathrm{EW}$ and $\mathrm{QCD}$ effects [67]. We recommend the latter procedure only for a 
SM-like Higgs boson. Contrary the inclusion of electroweak corrections due to light quarks [30,31], where the Higgs boson couples to either the $Z$ or $W^{ \pm}$boson, can be adjusted to the MSSM and accordingly the NMSSM in an appropriate way [68]. For this purpose the generalized couplings

$g_{V}^{H_{i}}=\mathcal{R}_{i 1}^{S} \cos \beta+\mathcal{R}_{i 2}^{S} \sin \beta$

of the $i$ th CP-even NMSSM Higgs boson to the heavy gauge boson $V \in\left\{W^{ \pm}, Z\right\}$ need to be inserted in the formulas of Ref. [7]. The missing projection $\mathcal{R}_{i 3}^{S}$ on the singlet component reflects the fact that the singlet does not couple to gauge bosons. The CP-odd Higgs bosons do not couple to gauge bosons either, such that electroweak corrections due to light quarks are absent.

\subsection{NLO virtual amplitude for gluon fusion}

Regarding the implementation of two-loop contributions to gluon fusion, we closely follow Refs. [36-38] for the MSSM, which can be translated to the NMSSM. Their calculation at NLO is based on an asymptotic expansion in the masses of the supersymmetric particles. We can project the form factors onto the ones in gauge eigenstates according to

$\mathcal{H}_{i}^{(2 l)}=-\frac{3}{4}\left(\mathcal{R}_{i 1}^{S} \mathcal{H}_{d}^{R,(2 l)}+\mathcal{R}_{i 2}^{S} \mathcal{H}_{u}^{R,(2 l)}+\mathcal{R}_{i 3}^{S} \mathcal{S}^{R,(2 l)}\right)$,

$\mathcal{A}_{i}^{(2 l)}=-\frac{3}{4}\left(\mathcal{R}_{i+1,2}^{P} \mathcal{H}_{A}^{I,(2 l)}+\mathcal{R}_{i+1,3}^{P} \mathcal{S}^{I,(2 l)}\right)$

The individual contributions in gauge eigenstates are presented in Sect. 2.4.1 for the CP-even and in Sect. 2.4.2 for the CP-odd Higgs bosons. We included the constant factor between Refs. [36-38] and our work in the above equations.

\subsubsection{CP-even Higgs bosons}

In this subsection we present the form factors for the CP-even Higgs bosons in gauge eigenstates ${ }^{1}$

$$
\begin{aligned}
\mathcal{H}_{d}^{R,(2 l)}= & \frac{1}{\sin \beta}\left[-m_{t} \mu s_{2 \theta_{t}} F_{t}^{2 l}+m_{Z}^{2} s_{2 \beta} D_{t}^{2 l}\right] \\
& +\frac{1}{\cos \beta}\left[m_{b} A_{b} s_{2 \theta_{b}} F_{b}^{2 l}+2 m_{b}^{2} G_{b}^{2 l}+2 m_{Z}^{2} c_{\beta}^{2} D_{b}^{2 l}\right]
\end{aligned}
$$

\footnotetext{
${ }^{1}$ In the CP-even sector we adapt the MSSM results of Refs. [36,38] to the NMSSM by isolating the terms proportional to the $H_{d}^{R} / H_{u}^{R}$ squark-squark couplings and replacing them by the $S^{R}$-squark-squark couplings for the form factor $\mathcal{S}$. Similarly we proceed in the CP-odd sector starting from the form factors of Ref. [37] taking into account the prerotation of the CP-odd Higgs mixing matrix.
}

$$
\begin{aligned}
\mathcal{H}_{u}^{R,(2 l)}= & \frac{1}{\cos \beta}\left[-m_{b} \mu s_{2 \theta_{b}} F_{b}^{2 l}-m_{Z}^{2} s_{2 \beta} D_{b}^{2 l}\right] \\
& +\frac{1}{\sin \beta}\left[m_{t} A_{t} s_{2 \theta_{t}} F_{t}^{2 l}+2 m_{t}^{2} G_{t}^{2 l}-2 m_{Z}^{2} s_{\beta}^{2} D_{t}^{2 l}\right] \\
\mathcal{S}^{R,(2 l)}= & \frac{1}{\sin \beta}\left[-\frac{1}{\sqrt{2}} m_{t} \lambda v_{d} s_{2 \theta_{t}} F_{t}^{2 l}\right] \\
& +\frac{1}{\cos \beta}\left[-\frac{1}{\sqrt{2}} m_{b} \lambda v_{u} s_{2 \theta_{b}} F_{b}^{2 l}\right]
\end{aligned}
$$

which includes the effective $\mu$ parameter defined in Eq. (4). All functions in $\mathcal{H}_{d}^{R,(2 l)}, \mathcal{H}_{u}^{R,(2 l)}$, and $\mathcal{S}^{R,(2 l)}$ can be directly taken over from Refs. [36,38], keeping in mind the different convention in the sign of the $\mu$ parameter. For on-shell (OS) parameters (see Refs. $[36,38]$ ) and thus for our implementation the contribution $F_{t}^{2 l}$ is shifted according to Section 3.3 of Ref. [38] and $F_{b}^{2 l}$ according to Ref. [36]. The shift also applies to $F_{t}^{2 l}$ and $F_{b}^{2 l}$ entering the singlet contribution $\mathcal{S}^{R}$, since the differences in the prefactors being $\mu, \lambda v_{d}$ or $\lambda v_{u}$ are not renormalized when taking into account SQCD contributions and therefore do not contribute to the described OS shifts.

It remains to discuss the inclusion of resummed sbottom contributions into the bottom-quark Yukawa coupling within the virtual corrections to gluon fusion, where care has to be taken to avoid a double-counting of NLO SQCD contributions. The naive resummation $\tilde{g}_{b}=g_{b} /\left(1+\Delta_{b}\right)$ is incorporated in the same way as in the case of the MSSM $[36,46]$. The resummation as presented in Sect. 2.2 instead needs the subtraction of the $\tan \beta$ enhanced contributions to $G_{b}^{2 l}$ multiplied with the corresponding coupling correction; in detail, for the three CP-even Higgs bosons $H_{i}$

$$
\begin{aligned}
& 2 m_{b}^{2} G_{b}^{2 l} \rightarrow 2 m_{b}^{2} G_{b}^{2 l} \\
& \quad-\frac{C_{F}}{2} \mathcal{G}_{1 / 2}^{1 l}\left(\tau_{b}\right) \mu \tan \beta\left(-m_{\tilde{g}} I\left(m_{\tilde{b}_{1}}^{2}, m_{\tilde{b}_{2}}^{2}, m_{\tilde{g}}^{2}\right)\right) K_{i}^{\prime}
\end{aligned}
$$

with the factor $K_{i}^{\prime}$ being

$$
K_{i}^{\prime}=\frac{1}{1+\Delta_{b}}\left[1-\left(\frac{\mathcal{R}_{i 2}^{S}}{\mathcal{R}_{i 1}^{S} \tan \beta}+\frac{\mathcal{R}_{i 3}^{S} v \cos \beta}{\mathcal{R}_{i 1}^{S} v_{s}}\right)\right],
$$

with $i \in\{1,2,3\}$.

All occurrences of the bottom-quark Yukawa coupling in the two-loop amplitude are multiplied with the factor $K_{i}=$ $\tilde{g}_{b}^{H_{i}} / g_{b}^{H_{i}}$ using Eq. (10), such that the shift reported in Eq. (19) avoids double-counting of the purely SQCD-induced contributions at the two-loop level. Employing the expansion in heavy SUSY masses the NLO virtual contributions to neutral CP-even Higgs production in the NMSSM are now fully presented. 


\subsubsection{CP-odd Higgs bosons}

We now turn to the case of the two CP-odd Higgs bosons, where we present the form factor in the basis $H^{\prime I}$ after a prerotation from gauge eigenstates ${ }^{1}$. At LO only diagrams involving quarks coupling to the pseudoscalar $A$ exist, such that the form factor at $\mathrm{LO}$ only consists of the part $\mathcal{H}_{A}^{I,(1 l)}$, whereas $\mathcal{S}^{I,(1 l)}$ equals zero. At NLO, however, couplings of $A$ and $S^{I}$ to squarks $\tilde{q}_{i} \tilde{q}_{j}$ for $i \neq j$ induce contributions to Higgs production. The two-loop form factor presented in Ref. [37] for the MSSM can therefore be translated to

$$
\begin{aligned}
& \mathcal{H}_{A}^{I,(2 l)}=\left[\cot \beta\left(\mathcal{K}_{t g}^{2 l}+\mathcal{K}_{t \tilde{t} \tilde{g}}^{2 l}\right)+\tan \beta\left(\mathcal{K}_{b g}^{2 l}+\mathcal{K}_{b \tilde{b} \tilde{g}}^{2 l}\right)\right] \\
& \mathcal{S}^{I,(2 l)}=\left[\cot \beta \mathcal{K}_{t \tilde{g} \tilde{g}}^{S, 2 l}+\tan \beta \mathcal{K}_{b \tilde{b} \tilde{g}}^{S, 2 l}\right] .
\end{aligned}
$$

Whereas the individual contributions to $\mathcal{H}_{A}^{I,(2 l)}$ can be taken from Ref. [37], we present the contributions to $\mathcal{S}^{I,(2 l)}$ separately:

$$
\begin{aligned}
\mathcal{K}_{t \tilde{t} \tilde{g}}^{S, 2 l}= & \frac{C_{F}}{2} \mathcal{K}^{1 l}\left(\tau_{t}\right) \frac{m_{\tilde{g}}}{m_{t}} \frac{m_{t} \frac{1}{\sqrt{2}} \lambda v}{m_{\tilde{t}_{1}}^{2}-m_{\tilde{t}_{2}}^{2}} \\
& \times\left(\frac{x_{1}^{t}}{1-x_{1}^{t}} \ln x_{1}^{t}-\frac{x_{2}^{t}}{1-x_{2}^{t}} \ln x_{2}^{t}\right)-\frac{m_{t}}{m_{\tilde{g}}} S_{2 \theta_{t}} \mathcal{R}_{1}^{\prime t} \\
& +\frac{2 m_{t}^{2} \frac{1}{\sqrt{2}} \lambda v}{m_{\tilde{g}}\left(m_{\tilde{t}_{1}}^{2}-m_{\tilde{t}_{2}}^{2}\right)} \mathcal{R}_{2}-\frac{1}{2} \mathcal{K}^{1 l}\left(\tau_{t}\right) \frac{m_{A_{i}}^{2}}{m_{\tilde{t}_{1}}^{2}-m_{\tilde{t}_{2}}^{2}} \mathcal{R}_{4}^{\prime t}, \\
\mathcal{K}_{b \tilde{b} \tilde{g}}^{S, 2 l}= & \frac{C_{F}}{2} \mathcal{K}^{1 l}\left(\tau_{b}\right) \frac{m_{\tilde{g}}}{m_{b}} \frac{m_{b} \frac{1}{\sqrt{2}} \lambda v}{m_{\tilde{b}_{1}}^{2}-m_{\tilde{b}_{2}}^{2}} \\
& \times\left(\frac{x_{1}^{b}}{1-x_{1}^{b}} \ln x_{1}^{b}-\frac{x_{2}^{b}}{1-x_{2}^{b}} \ln x_{2}^{b}\right)-\frac{m_{b}}{m_{\tilde{g}}} s_{2 \theta_{b}} \mathcal{R}_{1}^{\prime b}
\end{aligned}
$$

with $x_{i}^{t}=m_{\tilde{t}_{i}}^{2} / m_{\tilde{g}}^{2}$ and $x_{i}^{b}=m_{\tilde{b}_{i}}^{2} / m_{\tilde{g}}^{2}$. The functions $\mathcal{K}^{1 l}$ and $\mathcal{R}_{2}$ can be found in Ref. [37]. The functions $\mathcal{R}^{\prime t}{ }_{1}$ and $\mathcal{R}^{\prime t}$ are given by

$$
\begin{aligned}
\mathcal{R}^{\prime t}= & \frac{C_{F}}{\left(x_{1}^{t}-x_{2}^{t}\right)^{2}} \frac{\frac{1}{\sqrt{2}} \lambda v}{m_{\tilde{g}}}\left(1+\frac{1}{2} \mathcal{K}^{1 l}\left(\tau_{t}\right)\right) \\
& \times\left[\frac{x_{1}^{t 2}\left(1-2 x_{2}^{t}\right)}{2\left(1-x_{1}^{t}\right)\left(1-x_{2}^{t}\right)}+\frac{x_{1}^{t}\left(x_{1}^{t 2}-2 x_{2}^{t}+x_{1}^{t} x_{2}^{t}\right) \ln x_{1}^{t}}{2\left(1-x_{1}^{t}\right)^{2}}\right] \\
& -\left(x_{1}^{t} \leftrightarrow x_{2}^{t}\right), \\
\mathcal{R}_{4}^{\prime t}= & \frac{C_{F}}{\left(x_{1}^{t}-x_{2}^{t}\right)^{2}} \frac{\frac{1}{\sqrt{2}} \lambda v}{m_{\tilde{g}}} \\
& \times\left[\frac{x_{1}^{t 2}\left(1-2 x_{2}^{t}\right)}{2\left(1-x_{1}^{t}\right)\left(1-x_{2}^{t}\right)}+\frac{x_{1}^{t}\left(x_{1}^{t 2}-2 x_{2}^{t}+x_{1}^{t} x_{2}^{t}\right) \ln x_{1}^{t}}{2\left(1-x_{1}^{t}\right)^{2}}\right] \\
& -\left(x_{1}^{t} \leftrightarrow x_{2}^{t}\right) .
\end{aligned}
$$

In the bottom sector the relevant function yields

$$
\begin{aligned}
\mathcal{R}_{1}^{\prime b}= & \frac{C_{F}}{\left(x_{1}^{b}-x_{2}^{b}\right)^{2}} \frac{\frac{1}{\sqrt{2}} \lambda v}{m_{\tilde{g}}} \\
& \times\left[\frac{x_{1}^{b 2}\left(1-2 x_{2}^{b}\right)}{2\left(1-x_{1}^{b}\right)\left(1-x_{2}^{b}\right)}+\frac{x_{1}^{b}\left(x_{1}^{b 2}-2 x_{2}^{b}+x_{1}^{b} x_{2}^{b}\right) \ln x_{1}^{b}}{2\left(1-x_{1}^{b}\right)^{2}}\right] \\
& -\left(x_{1}^{b} \leftrightarrow x_{2}^{b}\right) .
\end{aligned}
$$

The shifts of individual contributions in the case of OS parameters can be taken over from the MSSM case. The inclusion of resummed sbottom contributions to the bottomquark Yukawa coupling needs the following shift in the twoloop form factor for the CP-odd Higgs bosons $A_{i}$ :

$\mathcal{K}_{b \tilde{b} \tilde{g}}^{2 l} \rightarrow \mathcal{K}_{b \tilde{b} \tilde{g}}^{2 l}-\frac{C_{F}}{2} \mathcal{K}^{1 l}\left(\tau_{b}\right) \mu \tan \beta\left(-m_{\tilde{g}} I\left(m_{\tilde{b}_{1}}^{2}, m_{\tilde{b}_{2}}^{2}, m_{\tilde{g}}^{2}\right)\right) K_{i}^{\prime}$

using

$$
K_{i}^{\prime}=\frac{1}{1+\Delta_{b}}\left[1+\left(\frac{1}{\tan ^{2} \beta}+\frac{\mathcal{R}_{i+1,3}^{P} v}{\mathcal{R}_{i+1,2}^{P} v_{s} \tan \beta}\right)\right],
$$

with $i \in\{1,2\}$.

Again we point out that our sign convention with respect to $\mu$ is opposite to Ref. [37] and all occurrences of the bottomquark Yukawa coupling in the two-loop amplitude are multiplied with the factor $K_{i}=\tilde{g}_{b}^{A_{i}} / g_{b}^{A_{i}}$ using Eq. (11).

\subsection{Bottom-quark annihilation cross section in the 5FS}

The generalization of the calculation of bottom-quark annihilation cross sections in the five-flavor scheme (5FS) from the MSSM to the NMSSM case is straightforward by using the appropriate couplings of the Higgs bosons to the bottom quarks. For this purpose the resummation of the sbottom contributions as described in Sect. 2.2 is taken into account. For the specific case of the singlet-like Higgs boson we point out that in the case that the coupling to the bottom quark vanishes (due to cancellations in the mixing with the Higgs doublets) a priori the coupling to sbottom squarks can still be present. This is not taken into account by the resummation procedure.

\section{Implementation in SusHi}

In the current implementation of neutral Higgs production in the real NMSSM within the code SusHi the Higgs mixing matrices as well as the Higgs masses have to be provided as input in SUSY Les Houches Accord 
( SLHA) form [69, 70] and can be obtained by spectrum generators for the NMSSM. Common codes are NMSSMTOols [71-74], NMSSMCALC [57,65,75-77], SOFTSUSY [78,79], SPheno+Sarah [80-82] and FlexibleSUSY+Sarah [80,83].

Special attention needs to be paid to the renormalization of the stop and sbottom sector, which in the ideal form should be identical in the calculation of Higgs masses and mixing and the calculation of Higgs production cross sections. For the time being, SusHi either relies on the internal calculation of on-shell stop and sbottom sectors as described in the manual [7] or on the specification of the on-shell masses $m_{\tilde{q}_{1}}$ and $m_{\tilde{q}_{2}}$ and mixing angles $\theta_{\tilde{q}}$ in the input file. For both cases input files can be found in the folder / example within the SusHi tarball. The user is asked to check the meaning of output parameters of spectrum generators, i.e. the chosen renormalization scheme. If the user specifies the on-shell squark masses and mixing angles together with the on-shell soft-breaking parameters $A_{t}$ and $A_{b}$ by hand, she/he should make sure that in the stop sector $A_{t}$ as well as the on-shell top-quark mass $m_{t}$, the on-shell stop masses $m_{\tilde{t}_{1}}$ and $m_{\tilde{t}_{2}}$ and the mixing angle $\theta_{\tilde{t}}$ fit the formula

$\sin \left(2 \theta_{\tilde{t}}\right)=\frac{2 m_{t}\left(A_{t}-\mu / \tan \beta\right)}{m_{\tilde{t}_{1}}^{2}-m_{\tilde{t}_{2}}^{2}}$.

In the sbottom sector, on-shell and tree-level masses on the other hand differ by a shift in the $(1,1)$-element; see $\Delta M_{L}^{2}$ in Ref. [7]. Moreover, we employ the scheme which works with a dependent bottom-quark mass $m_{b}$, whereas $A_{b}$ is defined to be on-shell; see e.g. Refs. [84-86]. To allow for maximal flexibility the specification of on-shell squark masses and mixing angles is now also possible in the case of the MSSM. The Block RENORMSBOT is not of relevance in such input files, since $m_{b}$ is chosen as dependent parameter, whereas the squark mixing angle $\theta_{b}$ and the soft-breaking parameter $A_{b}$ are understood as renormalized on-shell.

Two options for the pseudoscalar Higgs mixing matrix are accepted as input by SusHi, namely the full Higgs mixing matrix, which corresponds to the multiplication $\mathcal{R}^{P} \mathcal{R}^{G}$ in the above notation, but instead also the rotation matrix $\mathcal{R}^{P}$ can be used as input. Following SLHA2 [70] the full matrix $\left(\mathcal{R}^{P} \mathcal{R}^{G}\right)_{i j}$ is provided in Block NMAMIX and asks for entries $i j$ with $i \in\{2,3\}$ and $j \in\{1,2,3\}$. The matrix $\mathcal{R}_{i j}^{P}$ can be specified in Block NMAMIXR, which only asks for entries $i j$ with $\{i, j\} \in\{2,3\}$. We point out that in contrast to other codes the Goldstone boson remains the first mass eigenstate, such that Block NMAMIXR does not ask for entries with $i=1$ or $j=1$. The elements of the CP-even Higgs boson mixing matrix are specified in Block NMHMIX [70]. The Higgs masses need to be given in Block MASS using entries 25, 35 and 45 for the CP-even Higgs bosons and 36, 46 for the CP-odd Higgs bosons.
The block Block EXTPAR still contains the gluino mass as well as the soft-breaking parameters for the third generation squark sector. Entry 23 for the $\mu$ parameter is, however, replaced by entry 65 , where the effective value of $\mu$ needs to be specified. Moreover, entry 61 asks for the choice of $\lambda$. SusHi extracts the VEV $v_{s}$ from $\mu$ and $\lambda$. Since the Higgs sectors including their mixing are provided, there is no need to provide the parameters $\kappa, A_{\kappa}, A_{\lambda}$ (or $m_{H^{ \pm}}$) in the SusHi input, since they do not enter the couplings relevant for Higgs production. The Block SUSHI entry 2 specifies the Higgs boson, for which cross sections are requested. The $\mathrm{CP}$-even Higgs bosons are numbered 11, 12, and 13, the CP-odd Higgs bosons 21 and 22. Similarly the options 11, 12, and 21 also work in the 2-Higgs-Doublet Model (2HDM) and the MSSM and 11 and 21 in the SM. A CP-odd Higgs boson 21 in the $\mathrm{SM}$ is obtained from the $2 \mathrm{HDM}$ case with $\tan \beta=1$. We note that SusHi is still compatible with input files with 0 (light Higgs), 1 (pseudoscalar) and 2 (heavy Higgs) as options for entry 2. Output files, however, stick to the new convention.

For the time being we emphasize that $\mathrm{SusHi}$ is not strictly suitable for very low values of Higgs masses $m_{\phi}<20 \mathrm{GeV}$, where quark threshold effects start to become relevant and also electroweak corrections are not implemented. This statement mostly applies to studies of a very light CP-odd Higgs boson, which is poorly constrained by LEP experiments in contrast to a light CP-even Higgs boson [87].

\section{Phenomenological study}

In this section we elaborate on the phenomenological consequences of the additional $\mathrm{SU}(2)_{L}$ singlet in the NMSSM with respect to the MSSM for neutral Higgs production. Neglecting the squark-induced contributions to gluon fusion, the only consequence of the additional singlet component is another admixture of the three CP-even/two CP-odd Higgs bosons. However, no generically new contributions to Higgs boson production arise. This differs when taking into account squark-induced contributions to gluon fusion due to the additional singlet to squark couplings. In particular for the $\mathrm{CP}$ odd Higgs bosons squark contributions are only induced at the two-loop level due to the non-diagonal structure of the CP-odd Higgs bosons to squark couplings. Subsequently we work with two scenarios, start with their definition, present the Higgs boson masses and admixtures, and then discuss the behavior of cross sections, including the squark and electroweak corrections to the gluon-fusion cross section. Our studies are performed for a proton-proton collider with a center-of-mass (cms) energy of $\sqrt{s}=13 \mathrm{TeV}$, as planned for the second run of the LHC. Lastly we add a short discussion of renormalization and factorization scale uncertainties as well as PDF $+\alpha_{s}$ uncertainties for one of the two scenarios. 


\subsection{Scenarios $S_{1}$ and $S_{2}$}

To present the most relevant features of the NMSSM for what concerns neutral Higgs production we pick two scenarios. The first scenario $S_{1}$ is in the vicinity of the natural NMSSM [5] with a rather large value of $\lambda=0.62$. Other input parameters are $M_{1}=150 \mathrm{GeV}, M_{2}=340 \mathrm{GeV}$, $M_{3}=1.5 \mathrm{TeV}, \tan \beta=2, A_{\kappa}=-20 \mathrm{GeV}$ and $\mu=$ $200 \mathrm{GeV} . A_{\lambda}$ is determined from the charged Higgs mass $m_{H^{ \pm}}=400 \mathrm{GeV}$. The size of $\lambda$ ensures a large mixing of the singlet component with the $H_{d}^{0}$ and $H_{u}^{0}$ doublets. All soft-breaking masses are set to $1.5 \mathrm{TeV}$ except for the soft-breaking masses of the third generation squark sector, which are fixed to $750 \mathrm{GeV}$. The soft-breaking couplings are set to $A=1.8 \mathrm{TeV}$. The on-shell stop masses are then given by $m_{\tilde{t}_{1}}=544.7 \mathrm{GeV}$ and $m_{\tilde{t}_{2}}=941.2 \mathrm{GeV}$, whereas the sbottom masses are $m_{\tilde{b}_{1}}=749.4 \mathrm{GeV}$ and $m_{\tilde{b}_{2}}=757.4 \mathrm{GeV}$. We vary $\kappa$ between 0.15 and 0.80 and thus vary the mass of the singlet-like Higgs component in particular in the CP-even Higgs sector. We note that for illustrative reasons the perturbativity limit approximately given by $\sqrt{\lambda^{2}+\kappa^{2}}<0.7$ is not always fulfilled in our study. We work out the characteristics for the singlet-like component in the following discussion. The relevant input for SusHi is obtained with NMSSMCALC 1.03, which incorporates the leading two-loop corrections $\mathcal{O}\left(\alpha_{s} \alpha_{t}\right)$ to the Higgs boson masses calculated in the gaugeless limit with vanishing external momentum [77]. We request NMSSMCALC to work with an on-shell renormalized stop sector and add local modifications to the NMSSMCALC input routines to read in on-shell parameters rather than $\overline{\mathrm{DR}}$ renormalized parameters. ${ }^{2}$ These modifications guarantee identical on-shell stop masses in NMSSMCALC and SusHi. The renormalization of the sbottom sector on the other hand is performed SusHi-internally.

We also choose a second scenario $S_{2}$, in which we vary $\lambda$ to decouple the singlet-like Higgs from the Higgs doublets. The detailed choice of parameters is $M_{1}=150 \mathrm{GeV}, M_{2}=$ $300 \mathrm{GeV}, M_{3}=1.5 \mathrm{TeV}, \tan \beta=10, A=-2.0 \mathrm{TeV}, \kappa=$ $0.2, A_{\kappa}=-30 \mathrm{GeV}, \mu=130 \mathrm{GeV}$, and $m_{H^{ \pm}}=350 \mathrm{GeV}$. In this scenario we set the soft-breaking masses to $1.0 \mathrm{TeV}$. The on-shell stop and sbottom masses are given by $m_{\tilde{t}_{1}}=$ $824.1 \mathrm{GeV}, m_{\tilde{t}_{2}}=1173.4 \mathrm{GeV}, m_{\tilde{b}_{1}}=998.0 \mathrm{GeV}$ and $m_{\tilde{b}_{2}}=$ $1008.4 \mathrm{GeV}$. We vary $\lambda$ between 0.04 and 0.25 . For small values of $\lambda H_{1}$ corresponds to the SM-like Higgs boson with mass $m_{H_{1}} \sim 121 \mathrm{GeV}$. The lower bound at $\lambda=0.04$ is to avoid tiny cross sections for a heavy singlet-like Higgs boson and to keep its mass below the SUSY masses thresholds to justify the NLO SQCD expansion employed for the gluonfusion cross section calculation.

\footnotetext{
${ }^{2}$ We thank Kathrin Walz for instructions on how to modify the NMSSMCALC input routines.
}

Both our scenarios come along with rather light third generation squark masses at the low TeV scale. Contrary to the Higgs mass calculations the squark contributions completely decouple from Higgs production for heavy SUSY spectra. Our scenarios are chosen to flash the phenomenology of an additional singlet-like Higgs boson and thus do not always include a SM-like Higgs boson with mass $\sim 125 \mathrm{GeV}$ and are partially under tension from LEP searches [87] (for low CP-even Higgs masses below $110 \mathrm{GeV}$ ) or LHC searches [88-102].

We add for both scenarios the relevant SM input, which includes the $\overline{\mathrm{MS}}$ renormalized bottom-quark mass $m_{b}\left(m_{b}\right)=$ $4.20 \mathrm{GeV}$, which is translated into a bottom-quark pole mass of $m_{b}=4.92 \mathrm{GeV}$. In SusHi we choose the renormalization scheme, where the bottom-quark pole mass enters all occurrences of heavy bottom-quark masses in the loops and the bottom-quark Yukawa coupling for the gluon-fusion cross section. Bottom-quark annihilation is based on the running $\overline{\mathrm{MS}}$ renormalized bottom-quark Yukawa coupling. As pointed out in Ref. [46] the gluon densities are hardly dependent on the bottom-quark pole mass fit value of the PDF fitting groups, emphasizing that there is no need to adjust the bottom-quark pole mass to the PDF fit value for the calculation of the gluon fusion cross section. The top-quark pole mass equals $m_{t}=173.3 \mathrm{GeV}$. The strong coupling constant $\alpha_{s}\left(m_{Z}\right)$ is set to 0.1172 for the calculation of running masses, and is obtained from the corresponding PDF set for the cross section calculation. We choose MSTW2 008 [103] at the appropriate order in perturbation theory. Our central scale choices for gluon fusion are $m_{\phi} / 2$ for both renormalization and factorization scale, $\mu_{R}^{0}$ and $\mu_{F}^{0}$, respectively, and $\mu_{R}^{0}=m_{\phi}$ and $\mu_{F}^{0}=m_{\phi} / 4$ for bottom-quark annihilation.

\subsection{Higgs boson masses and singlet admixtures}

Subsequently we start with a discussion of the singlet admixture and the masses of the three CP-even and the two CP-odd Higgs bosons, which we obtain through a link to NMSSMCALC 1.03 as explained beforehand. For scenario $S_{1}$ the singlet component as a function of $\kappa$ for the three CPeven Higgs bosons is shown in Fig. 1a. Clearly, for low values of $\kappa$ the lightest Higgs $H_{1}$ is mainly singlet-like, whereas with increasing $\kappa$ the dominant singlet fraction moves from $H_{1}$ to $H_{2}$ and for large values of $\kappa$ to $H_{3}$. The sum of all singlet components yields $\sum_{i}\left|\mathcal{R}_{i 3}^{S}\right|^{2}=1$. The masses of the CPeven Higgs bosons can be found in Fig. 1b. With increasing $\kappa$ the mass term of the singlet component in gauge eigenstates is increasing proportional to $\kappa v_{s}$, such that the singlet-like Higgs boson can be identified with the Higgs boson linearly increasing in mass. Close to $\kappa \sim 0.35 \mathrm{H}_{2}$ shows the most dominant singlet fraction, which will later be visible in the gluon-fusion cross section. Scenario $S_{1}$ includes for $\kappa>0.3$ a SM-like Higgs boson $H_{1}$ with a mass of $m_{H_{1}} \sim 125 \mathrm{GeV}$. 
Fig. 1 a Singlet character and b masses of the three $\mathrm{CP}$-even Higgs bosons $H_{1}$ (black), $\mathrm{H}_{2}$ (red, dashed), $\mathrm{H}_{3}$ (blue, dot-dashed) as a function of $\kappa$ for scenario $S_{1}$ obtained from NMSSMCALC 1.03 ; $\mathbf{c}$ singlet character and $\mathbf{d}$ masses of the two CP-odd Higgs bosons $A_{1}$ (black), $A_{2}$ (red, dashed) as a function of $\kappa$ for scenario $S_{1}$ obtained from NMSSMCALC 1.03

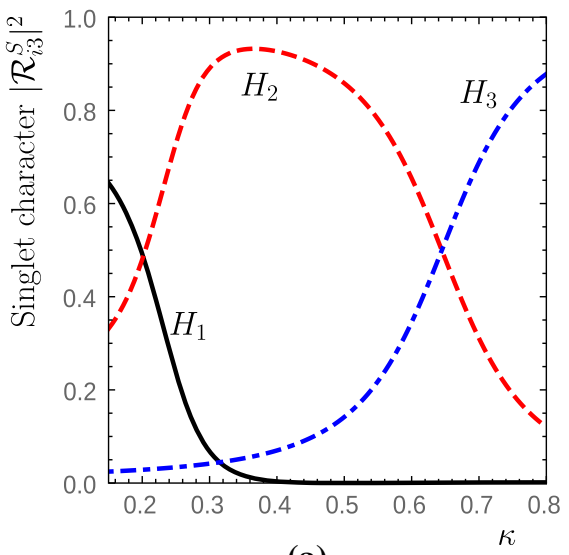

(a)

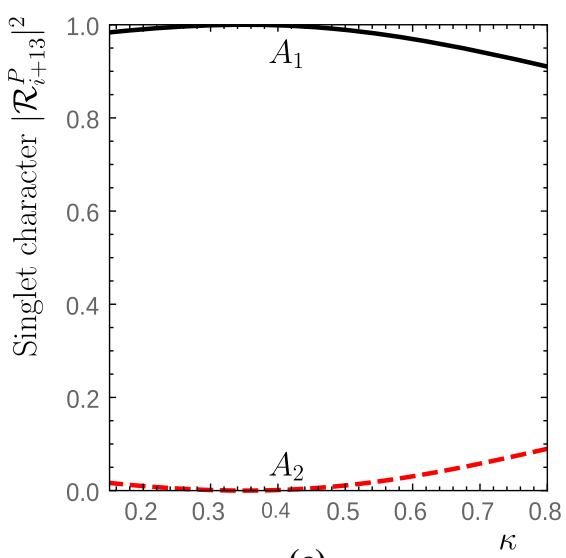

(c)

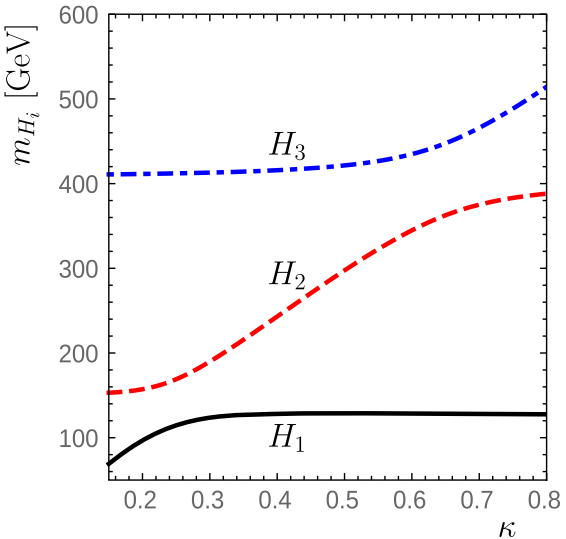

(b)

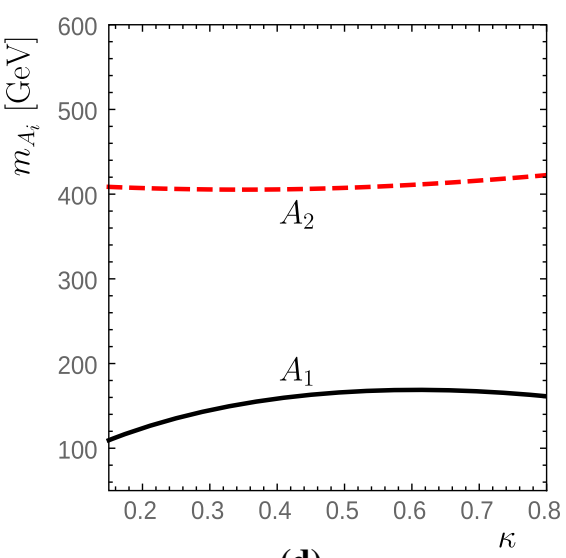

(d)
For very small values of $\kappa$ the decay $H_{2} \rightarrow H_{1} H_{1}$ opens and leaves a characteristic signature for the SM-like Higgs boson $\mathrm{H}_{2}$. Note that a light singlet-like Higgs boson lifts the mass of the SM-like CP-even Higgs through singlet-doublet mixing, which for our example equals $m_{H_{2}} \sim 153 \mathrm{GeV}$ for $\kappa=0.1$. The region of small $\kappa$ and a light CP-even singlet-like Higgs boson $H_{1}$ is largely constrained by the LEP experiments [87].

Figure 1c and d show the behavior of the singlet admixture and the masses for the two CP-odd Higgs bosons in scenario $S_{1}$ as a function of $\kappa$. We point again to the region in the vicinity of $\kappa \sim 0.35$, where the light CP-odd Higgs boson $A_{1}$ is a pure singlet-like CP-odd Higgs boson contrary to the CP-even Higgs boson $H_{2}$, for which $H_{d}^{R}$ and $H_{u}^{R}$ components remain. The coupling of $A_{1}$ to the quarks vanishes, but the coupling to the squarks is still present due to the relatively large value of $\lambda=0.62$, which will be apparent when calculating the gluon-fusion cross section.

For scenario $S_{2}$ Fig. 2 shows correspondingly the singlet character and masses for the $\mathrm{CP}$-even and $\mathrm{CP}$-odd Higgs bosons. Due to the fixed value of $\mu=\frac{1}{\sqrt{2}} \lambda v_{s}$ the singlet-like Higgs boson increases in mass (proportional to $\kappa v_{s}$ ) with decreasing $\lambda$ and thus for small $\lambda H_{3}$ as well as $A_{2}$ clearly decouple from the other Higgs bosons. We will later use this setup to show the decoupling behavior of the cross sections. Below $\lambda<0.05$ both $\mathrm{H}_{3}$ and $A_{2}$ have a singlet character, which exceeds $\left|\mathcal{R}_{33}^{S / P}\right|^{2}>0.999$.

\subsection{Scenario $S_{1}$ : inclusive cross sections for $\sqrt{s}=13 \mathrm{TeV}$}

In this subsection we investigate the gluon fusion $\sigma_{g g}$ and bottom-quark annihilation $\sigma_{b \bar{b}}$ cross sections for scenario $S_{1}$ for $\sqrt{s}=13 \mathrm{TeV}$ for a proton-proton collider. The subsequent statements are, however, hardly dependent on the $\mathrm{cms}$ energy and thus hold for the $7 / 8 \mathrm{TeV}$ LHC runs as well as for more energetic runs. Figure 3 shows the cross sections for the three CP-even Higgs bosons. Naturally the cross sections are strongly dependent on the Higgs mass, which are in turn a function of $\kappa$. Thus, the cross section for the second CP-even Higgs bosons $\mathrm{H}_{2}$ tends to decrease with increasing $\kappa$. Crucial is the singlet admixture of the Higgs boson under consideration. The larger the singlet component $\left|\mathcal{R}_{i 3}^{S}\right|^{2}$, the smaller the coupling to the quarks becomes and thus the more sensitive is the cross section to squark and electroweak contributions. For $\mathrm{H}_{2}$ we observe a cancellation of quark contributions through the admixtures with the $\mathrm{SU}(2)_{L}$ doublets around $\kappa \sim 0.35$, where in turn due to the generally small 
Fig. 2 a Singlet character and b masses of the three $\mathrm{CP}$-even Higgs bosons $H_{1}$ (black), $\mathrm{H}_{2}$ (red, dashed), $\mathrm{H}_{3}$ (blue, dot-dashed) as a function of $\lambda$ for scenario $S_{2}$ obtained from NMSSMCALC 1.03 ; c singlet character and $\mathbf{d}$ masses of the two CP-odd Higgs bosons $A_{1}$ (black), $A_{2}$ (red, dashed) as a function of $\lambda$ for scenario $S_{2}$ obtained from NMSSMCALC 1.03

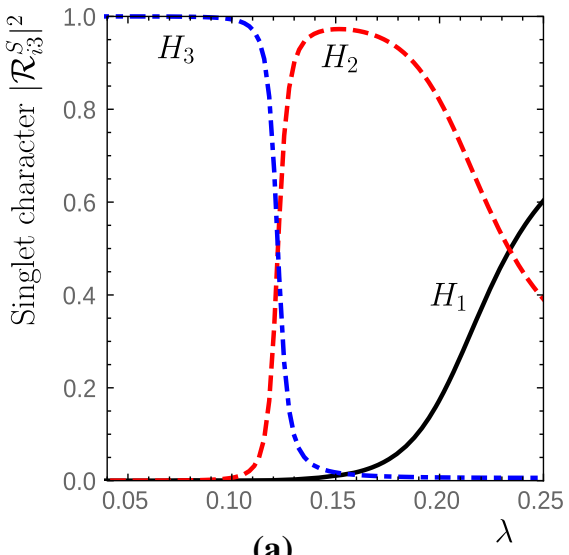

(a)
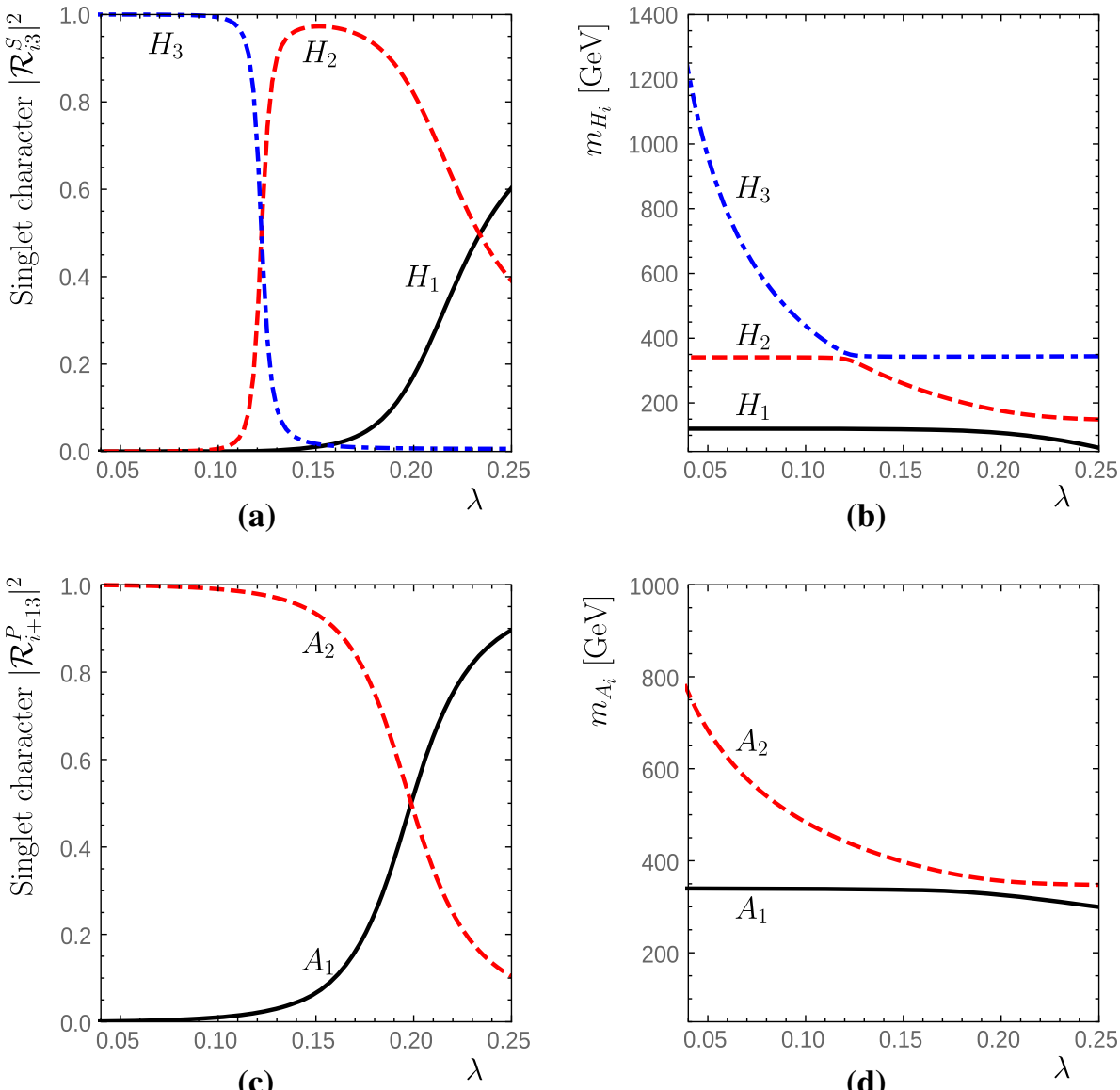

(c)

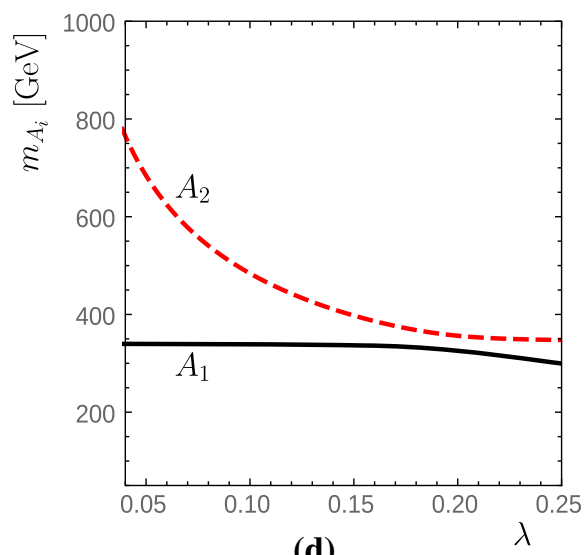

(d) cross section squark but also electroweak corrections to the gluon-fusion cross section are of large relevance; see Fig. 3c and d. For small values of $\kappa$ the decay $H_{2} \rightarrow H_{1} H_{1}$ opens in addition to the large gluon-fusion cross section for the singlet-like CP-even Higgs boson $H_{1}$. The region is therefore constrained by LEP experiments [87]. Much smoother is the behavior for the bottom-quark annihilation cross section, where the direct coupling to bottom quarks is related to the non-singlet character of the Higgs under consideration. In an interval around $\kappa \sim 0.35$ bottom-quark annihilation even exceeds the gluon-fusion cross section for $\mathrm{H}_{2}$ despite the small value of $\tan \beta=2$.

We show the effect of squark and electroweak contributions to gluon fusion for the three CP-even Higgs bosons in Fig. $3 \mathrm{c}$ and d. $\sigma_{g g}^{q+\tilde{q}}$ in Fig. $3 \mathrm{c}$ includes stop- and sbottomquark induced contributions at NLO SQCD on top of the quark-induced contributions without electroweak contributions and compares to the pure quark-induced cross section $\sigma_{g g}^{q}$ without electroweak contributions. All cross sections include NLO QCD quark contributions and the NNLO QCD top-quark induced contributions in the heavy top-quark effective theory. Figure $3 \mathrm{~d}$ accordingly shows the effect of electroweak contributions induced by light quarks following Eq. (15) in combination with Ref. [46] in comparison to the quark and squark induced cross section $\sigma_{g g}^{Q C D}=\sigma_{g g}^{q+\tilde{q}}$. Note that in all our figures $\sigma_{g g}$ corresponds to $\sigma_{g g}^{Q C D+\mathrm{EW}}$. As expected for $H_{2}$, the region with small quark contributions induced by the admixture with the $H_{d}^{R}$ and $H_{u}^{R}$ components is in particular sensitive to squark corrections. For the other Higgs bosons the squarks corrections in this scenario are incidentally all of the order of $\mathcal{O}(-10 \%)$ and mostly independent of $\kappa$. We note that the squark corrections are mainly induced by stop contributions, whereas sbottom-induced contributions only account for a small fraction. Interestingly, the squark contributions show an interference-like structure with a maximum and minimum around $\kappa \sim 0.35$, whereas the relative electroweak corrections are always positive. This can be understood from a sign change in the real part of the quark-induced LO and NLO amplitude for $H_{2}$ at $\kappa \sim 0.35$, which is of relevance for the squark contributions, whereas the imaginary part, more relevant for the electroweak contributions, does not change its sign. The size of the electroweak corrections for $\mathrm{H}_{2}$ follows from a suppression of the couplings of the second lightest Higgs $\mathrm{H}_{2}$ to the quarks in contrast to the couplings to gauge bosons. Obtaining a pure singlet-like Higgs boson in the CP-even Higgs sector, which neither couples to quarks nor to gauge bosons, rarely happens due to the mixing between both $S^{R}$ and $H_{d}^{R}$ as well as $S^{R}$ and $H_{u}^{R}$ for large val- 
Fig. 3 a Gluon fusion and b bottom-quark annihilation in $\mathrm{pb}$ at $\sqrt{s}=13 \mathrm{TeV}$ as well as $\mathbf{c}$ squark and $\mathbf{d}$ electroweak contributions to gluon fusion for the three CP-even Higgs bosons $H_{1}$ (black), $H_{2}$ (red, dashed), $\mathrm{H}_{3}$ (blue, dot-dashed) as a function of $\kappa$ for scenario $S_{1}$
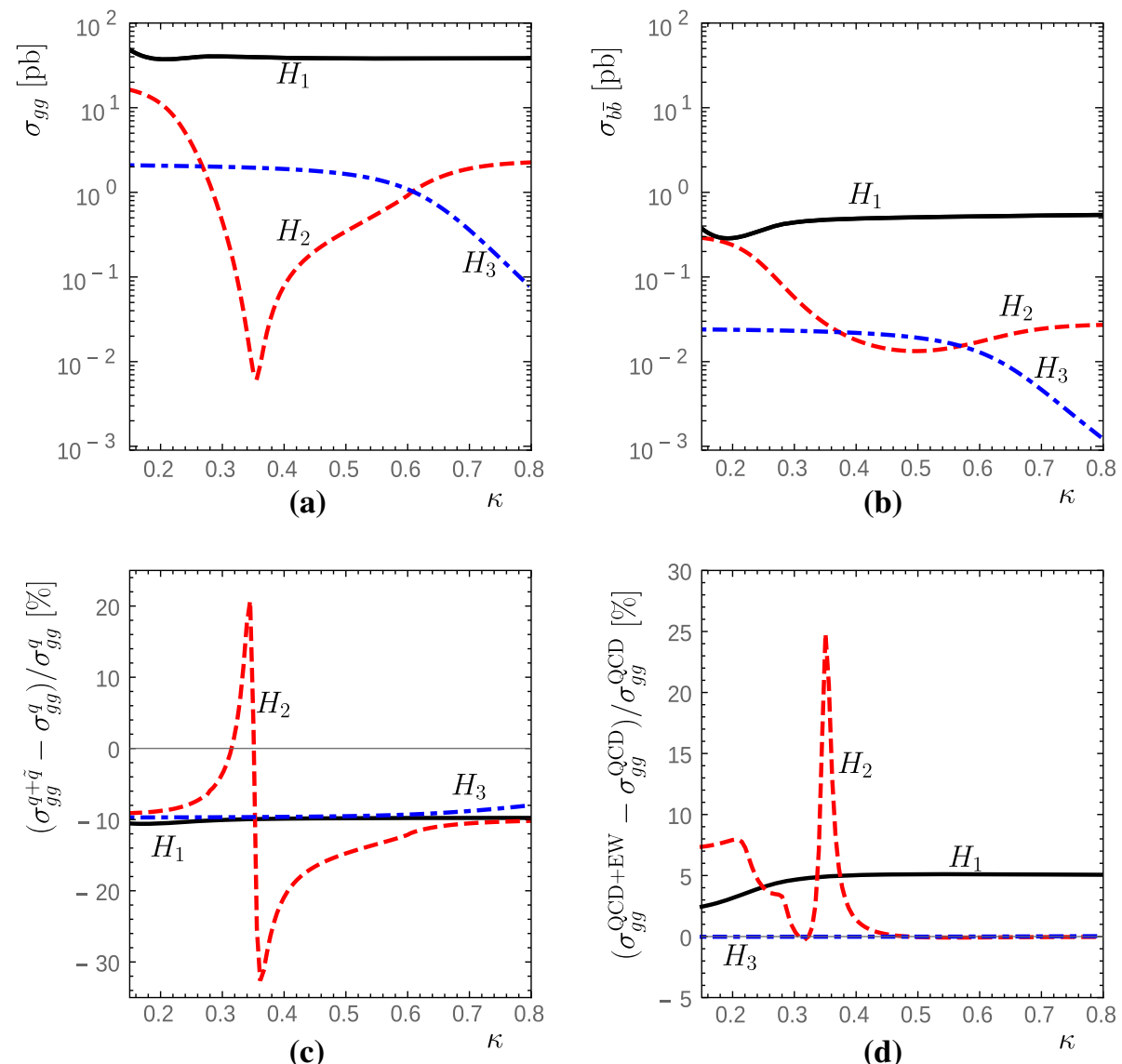

(d)

is unreliable, since SusHi calculates NLO QCD contributions through the multiplication of one-loop and two-loop contributions, where the latter tend to be significantly larger than the former and can thus even induce negative cross sections. However, in these regions the tiny cross sections are not of relevance for current searches. The cross section $\sigma_{g g}^{q+\tilde{q}}$ and the relative correction to the vanishing of only quark-induced cross sections of more than $100 \%$ need to be taken with care for the CP-odd Higgs bosons. The fact that for a pure singletlike CP-odd Higgs boson the gluon-fusion cross section at NLO SQCD completely vanishes due to the absence of a LO contribution motivates us to take into account NNLO SQCD stop contributions, as was done for the light CP-even Higgs boson in Ref. [46]. A first estimate yields tiny, positive cross sections, but we leave an inclusion in SusHi to future work. The CP-even Higgs bosons in contrast have a LO squark-induced contribution, which leaves $\sigma_{g g}^{q+\tilde{q}}$ mostly well behaved. Only in rare cases, where LO squark and quark contributions cancel, similar difficulties can arise.

\subsection{Scenario $S_{2}$ : inclusive cross sections for $\sqrt{s}=13 \mathrm{TeV}$}

In this subsection we examine the inclusive cross sections for scenario $S_{2}$, which includes low values of $\lambda$ and thus $\sigma_{g g}^{q+\tilde{q}}$ with squark-induced NLO SQCD contributions for $A_{1}$ 

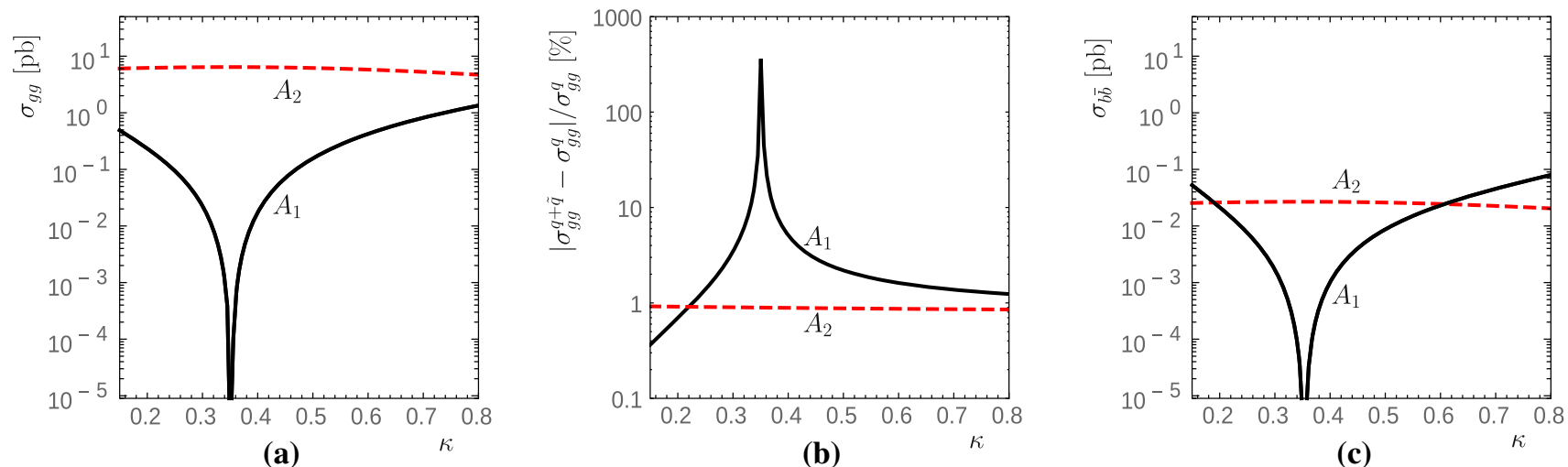

Fig. 4 a Gluon fusion and $\mathbf{c}$ bottom-quark annihilation in $\mathrm{pb}$ at $\sqrt{s}=13 \mathrm{TeV}$ as well as $\mathbf{b}$ absolute value of the relative squark corrections to gluon fusion for the two CP-odd Higgs bosons $A_{1}$ (black), $A_{2}$ (red, dashed) as a function of $\kappa$ for scenario $S_{1}$

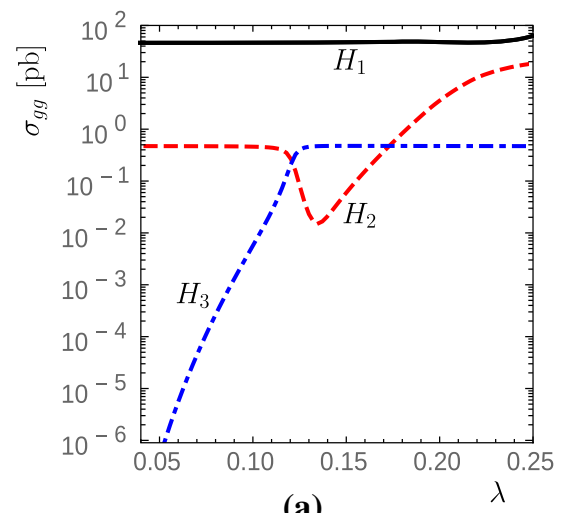

(a)

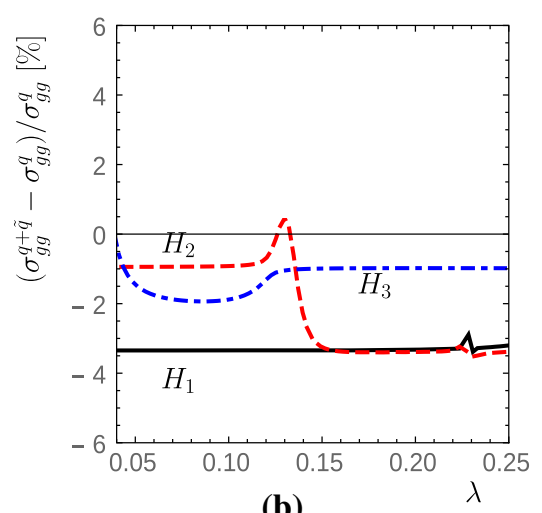

(b)

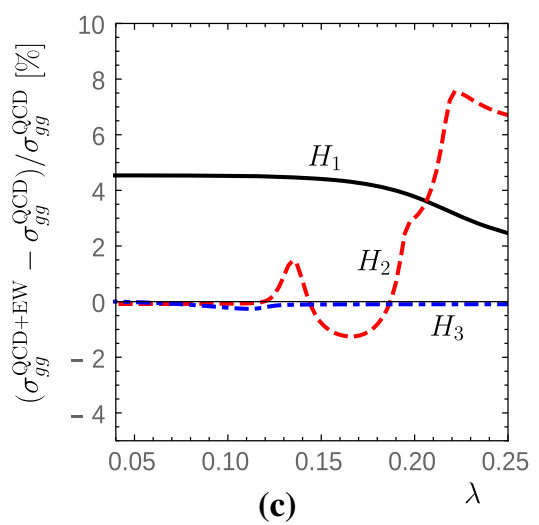

(c)

Fig. 5 a Gluon fusion in $\mathrm{pb}$ at $\sqrt{s}=13 \mathrm{TeV}$, b squark corrections and $\mathbf{c}$ electroweak corrections to gluon fusion for the three CP-even Higgs bosons $H_{1}$ (black), $H_{2}$ (red, dashed), $H_{3}$ (blue, dot-dashed) as a function of $\lambda$ for scenario $S_{2}$

reflects the decoupling of the singlet-like Higgs. The gluonfusion cross section together with squark contributions and electroweak corrections through light quarks are shown in Fig. 5 for the three CP-even Higgs bosons. With decreasing $\lambda$ the gluon-fusion cross section for $H_{2 / 3}$ rapidly decreases, which has two reasons: First the increasing mass naturally decreases the cross section, but second the singlet-like Higgs boson also decouples from the other two Higgs bosons-as apparent in Fig. 2. Thus, the indirect coupling to quarks is suppressed. Moreover, also the direct couplings to squarks are proportional to $\lambda$ and thus decrease in size with decreasing $\lambda$. The relative correction induced by squark contributions remains rather constant; see Fig. $5 b$. The small interference structure visible around $\lambda=0.22-0.23$ stems from the interchange of the dominant singlet character between $H_{1}$ and $H_{2}$. Remark that, for a SM Higgs, the decrease of the gluon-fusion cross section due to the increase in mass is between $m_{H}=500 \mathrm{GeV}$ and $m_{H}=1200 \mathrm{GeV}$ is only a factor of $\sim 47$, whereas we observe a decrease of more than five orders of magnitude, thus mainly driven by the decoupling. Electroweak corrections by light quarks as depicted in Fig. 5c are completely absent for a heavy singlet-like Higgs boson $H_{3}$, but they show a similar pattern at large $\lambda$ for $H_{2}$ as for small $\kappa$ in scenario $S_{1}$. The reason is that the Higgs mass $m_{H_{2}}$ in both scenarios crosses the $2 m_{Z}$ and $2 m_{W}$ thresholds for the electroweak corrections by light quarks. The bottomquark annihilation cross section shows a similar decoupling behavior and is thus not explicitly shown.

For the CP-odd Higgs bosons we show the corresponding decoupling limit in Fig. 6, where the gluon-fusion cross section and the bottom-quark annihilation cross section presented in Fig. 6a and c, respectively, decrease dramatically for the singlet-like Higgs bosons $A_{2}$. Again the squark corrections shown in Fig. $6 \mathrm{~b}$ remain rather constant. Given the fact that the total cross section for $A_{2}$ vanishes with decreasing $\lambda$, we can therefore conclude that both the indirect couplings to quarks for $A_{2}$ vanish, but also the direct couplings to the squarks vanish. Thus, the singlet-like Higgs boson $A_{2}$ decouples in the limit $\lambda$ being small.

We point out that the singlet-like Higgs bosons $\mathrm{H}_{3}$ and $A_{2}$ both approach the SUSY mass thresholds with decreasing $\lambda$. Therefore, we employ the lower bound of $\lambda=0.04$, since for lower values of $\lambda$ and thus larger masses of $H_{3}$ and $A_{2}$ we cannot guarantee the validity of the NLO SQCD 

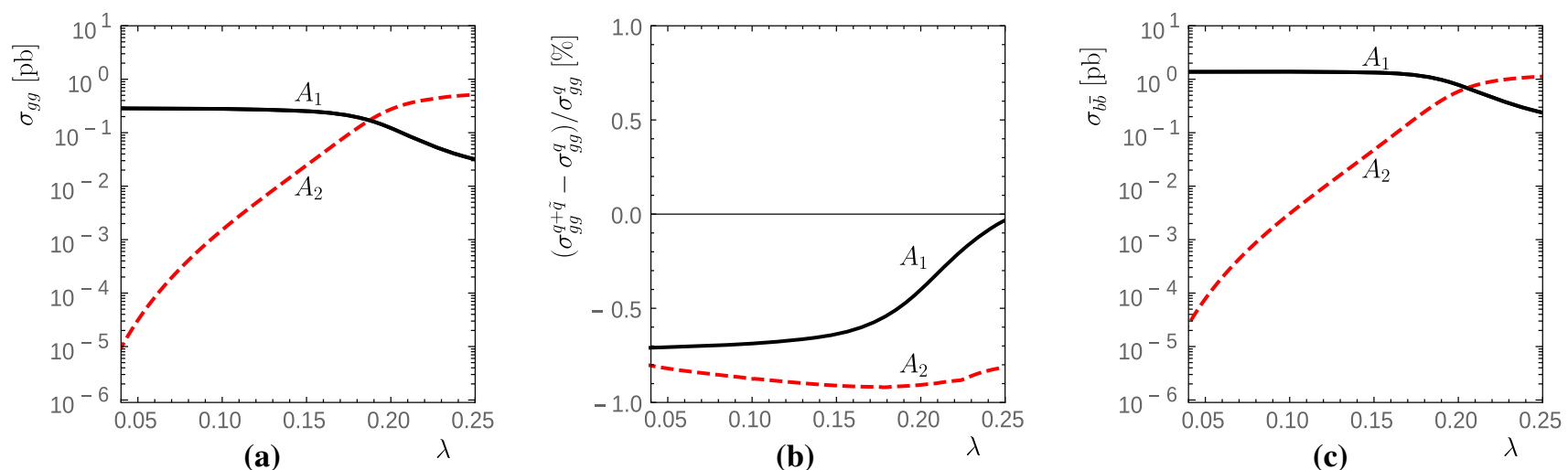

Fig. 6 a Gluon fusion and $\mathbf{c}$ bottom-quark annihilation in $\mathrm{pb}$ at $\sqrt{s}=13 \mathrm{TeV}$ as well as $\mathbf{b}$ the relative squark corrections to gluon fusion for the two CP-odd Higgs bosons $A_{1}$ (black), $A_{2}$ (red, dashed) as a function of $\lambda$ for scenario $S_{2}$

contributions implemented in SusHi. Reference [46] therefore assigned an additional theoretical uncertainty to the heavy SUSY masses expansion. In the decoupling regime we checked that the cross sections for $H_{1}, H_{2}$, and $A_{1}$ coincide with the MSSM cross sections obtained for a mixing angle of $\alpha=-0.12347$ with an accuracy of $\sim 10^{-4}$, which resembles the remaining singlet fraction of $H_{1}, H_{2}$, and $A_{1}$.

\subsection{Theory uncertainties}

In this section we briefly focus on theoretical uncertainties in the calculation of neutral Higgs boson production cross sections. Reference [46] identified the most important theory uncertainties for the MSSM, which mostly apply to our discussion of the NMSSM as well. Apart from the well-known renormalization and factorization scale and $\mathrm{PDF}+\alpha_{s}$ uncertainties for cross sections at a proton-proton collider an additional uncertainty for gluon-fusion cross section is the choice of a renormalization scheme for the bottom-quark Yukawa coupling, which is of particular relevance if the bottom-quark loop dominantly contributes. Secondly, the fact that NLO SQCD contributions are taken into account in an expansion of heavy SUSY masses induces an uncertainty, which grows for larger Higgs masses approaching SUSY particle masses thresholds. Thirdly, also relevant for bottom-quark annihilation, there are missing contributions in the resummation $\Delta_{b}$, which induce an uncertainty, in particular in the limit $\Delta_{b} \rightarrow-1$. All of the above theoretical uncertainties as discussed in Ref. [46] apply to the NMSSM in a similar way. In contrast to the MSSM, however, phenomenological studies of the NMSSM focus on lower values of $\tan \beta$, where both the uncertainty from the choice of the bottom-quark Yukawa coupling and the uncertainty induced from unknown contributions to $\Delta_{b}$ are of less importance. A detailed discussion in particular for the singlet-like $\mathrm{CP}$-even and CP-odd Higgs boson is left for future work.
In the following we stick to the commonly studied renormalization and factorization scale uncertainties as well as the PDF $+\alpha_{s}$ uncertainties. We present our results just for scenario $S_{1}$, since no generically new features appear in other SUSY scenarios. We start with the scale uncertainty, where we follow the prescription employed in Refs. $[8,46]$. We thus consider seven combinations of renormalization and factorization scales defined as set $C_{\mu}$ of pairs $\left(\mu_{R}, \mu_{F}\right)$ with $\mu_{R}=\left\{m_{\phi} / 4, m_{\phi} / 2, m_{\phi}\right\}$ and $\mu_{F}=\left\{m_{\phi} / 4, m_{\phi} / 2, m_{\phi}\right\}$ under the constraint $1 / 2 \leq \mu_{R} / \mu_{F} \leq 2$ for gluon fusion. For bottom-quark annihilation the set is determined from $\mu_{R}=\left\{m_{\phi} / 2, m_{\phi}, 2 m_{\phi}\right\}$ and $\mu_{F}=\left\{m_{\phi} / 8, m_{\phi} / 4, m_{\phi} / 2\right\}$ with the constraint $2 \leq \mu_{R} / \mu_{F} \leq 8$. The minimal and maximal cross sections are obtained according to

$$
\begin{aligned}
\sigma^{-} & :=\min _{\left(\mu_{R}, \mu_{F}\right) \in C_{\mu}}\left\{\sigma\left(\mu_{R}, \mu_{F}\right)\right\}, \\
\sigma^{+} & :=\max _{\left(\mu_{R}, \mu_{F}\right) \in C_{\mu}}\left\{\sigma\left(\mu_{R}, \mu_{F}\right)\right\},
\end{aligned}
$$

which we present relative to the cross sections $\sigma\left(\mu_{R}^{0}, \mu_{F}^{0}\right)$ at the central scales $\mu_{R}^{0}$ and $\mu_{F}^{0}$. They are $\mu_{R}^{0}=\mu_{F}^{0}=m_{\phi} / 2$ for gluon fusion and $\mu_{R}^{0}=m_{\phi}$ and $\mu_{F}^{0}=m_{\phi} / 4$ for bottom-quark annihilation. We therefore define the relative uncertainties

$\Delta_{\mu}^{+}:=\frac{\sigma^{+}-\sigma\left(\mu_{R}^{0}, \mu_{F}^{0}\right)}{\sigma\left(\mu_{R}^{0}, \mu_{F}^{0}\right)}, \quad \Delta_{\mu}^{-}:=\frac{\sigma^{-}-\sigma\left(\mu_{R}^{0}, \mu_{F}^{0}\right)}{\sigma\left(\mu_{R}^{0}, \mu_{F}^{0}\right)}$.

The scale uncertainties are shown for both the CP-even and the CP-odd Higgs bosons in Fig. 7 for $\sqrt{s}=13 \mathrm{TeV}$. In case of gluon fusion the SM-like Higgs comes with a scale uncertainty of about $\mathcal{O}( \pm 10 \%)$ taking into account NNLO QCD top-quark contributions in the heavy top-quark effective theory. The scale uncertainty is naturally strongly dependent on the individual contributions to the cross section and increases in particular in regions, where the top-quark induced contributions are small or quark contributions to the 
Fig. 7 Scale uncertainties $\Delta_{\mu}^{+}$ and $\Delta_{\mu}^{-}$for $\mathbf{a}, \mathbf{c}$ gluon fusion and $\mathbf{b}, \mathbf{d}$ bottom-quark annihilation for $\mathbf{a}, \mathbf{b}$ the three CP-even Higgs bosons $H_{1}$ (black), $\mathrm{H}_{2}$ (red, dashed), $\mathrm{H}_{3}$ (blue, dot-dashed) and for $\mathbf{c}, \mathbf{d}$ the two CP-odd Higgs bosons $A_{1}$ (black), $A_{2}$ (red, dashed) as a function of $\kappa$ for scenario $S_{1}$

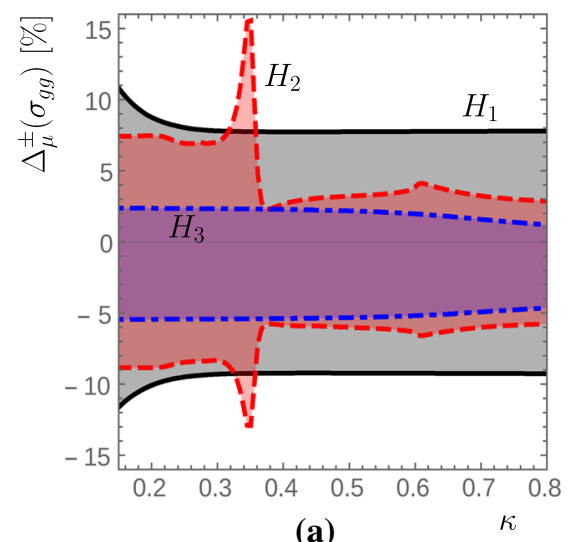

(a)

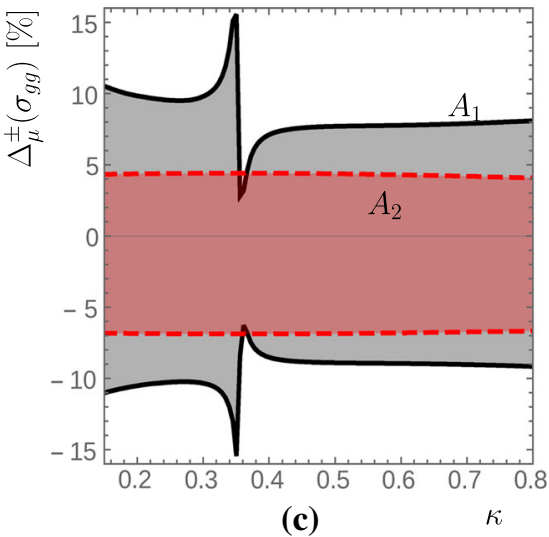

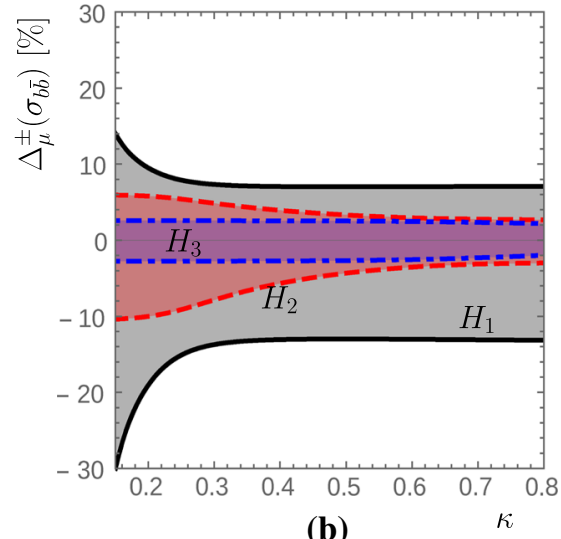

(b)

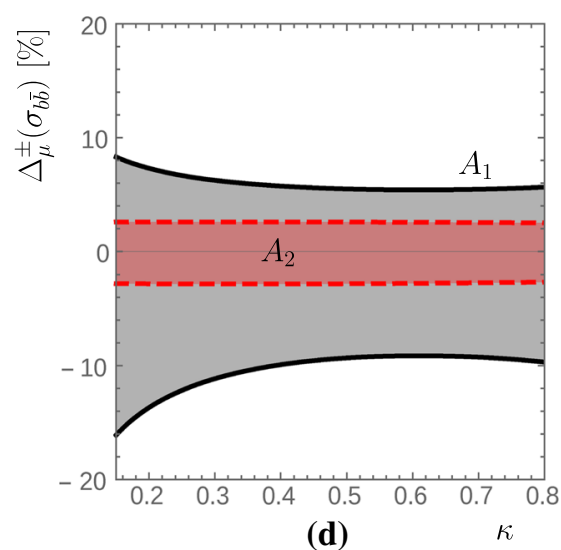

gluon fusion cross section cancel and come along with large squark and/or electroweak corrections. The latter effect is very pronounced for $H_{2}$ in Fig. 7a and for $A_{1}$ in Fig. 7c.

For bottom-quark annihilation as shown in Fig. 7b and d the scale uncertainty is mainly dependent on the Higgs mass, rather than the specific SUSY scenario. The large uncertainty for low Higgs masses reflects the need to move toward the four-flavor scheme (4FS) $[104,105]$ in the description of the process.

As a last step we discuss PDF $+\alpha_{s}$ uncertainties by applying the practical PDF4LHC recommendation $[106,107]$ for the MSTW2 008 [103] PDF sets in order to emphasize the findings of Ref. [46] for the case of the NMSSM. For this purpose we combine the results obtained with the 41 PDF sets of MSTW2008(n) nlo68cl with the $\alpha_{s}$ uncertainties obtained by the PDF sets, which vary $\alpha_{s}$ within the $68 \%$ confidence level interval. Figure 8 shows the $\mathrm{PDF}+\alpha_{s}$ relative uncertainties $\Delta_{\mathrm{PDF}+\alpha_{\mathrm{s}}}^{ \pm}$with respect to the standard PDF $+\alpha_{s}$ choice for the three CP-even Higgs bosons for gluon fusion and bottom-quark annihilation. The standard PDF $+\alpha_{s}$ choice equals the zeroth PDF set of MSTW2008(n) nlo68cl together with the standard values $\alpha_{s}=0.120$ at NLO and $\alpha_{s}=0.117$ at NNLO QCD. Similar to the MSSM the uncertainties are mainly dependent on the Higgs mass and only slightly dependent on the specific
SUSY scenario, even for the singlet-like Higgs boson. A very similar result applies to the CP-odd Higgs sector and is thus not explicitly presented. It therefore seems sufficient to take over the full relative $\mathrm{PDF}+\alpha_{s}$ uncertainties from a CP-even or CP-odd SM Higgs boson with the same mass, which is easily adjustable to future updates of the PDF4LHC recommendation. Taking into account the combination of the newest MMHT2 014 [108], NNPDF 3.0 [109] and CT1 0 [110] PDF sets naturally results in larger PDF $+\alpha_{s}$ uncertainties. However, the simple recipe to obtain $\mathrm{PDF}+\alpha_{s}$ uncertainties just as a function of the Higgs mass is applicable for the combination of the PDF sets provided by the PDF fitting groups as well.

\section{Conclusions}

We presented accurate predictions for neutral Higgs boson production at proton colliders through gluon fusion and bottom-quark annihilation in the CP-conserving NMSSM. For gluon fusion we adapt the full NLO QCD and SQCD results from the MSSM to the NMSSM, based on an asymptotic expansion in heavy SUSY masses for squark and squark/quark/gluino two-loop contributions. Top-quark induced NNLO QCD contributions are added in the heavy 
Fig. $8 \mathrm{PDF}+\alpha_{s}$ uncertainties for $\mathbf{a}$ gluon fusion and $\mathbf{b}$ bottom-quark annihilation for the three CP-even Higgs bosons $H_{1}$ (black), $H_{2}$ (red, dashed), $\mathrm{H}_{3}$ (blue, dot-dashed) as a function of $\kappa$ for scenario $S_{1}$

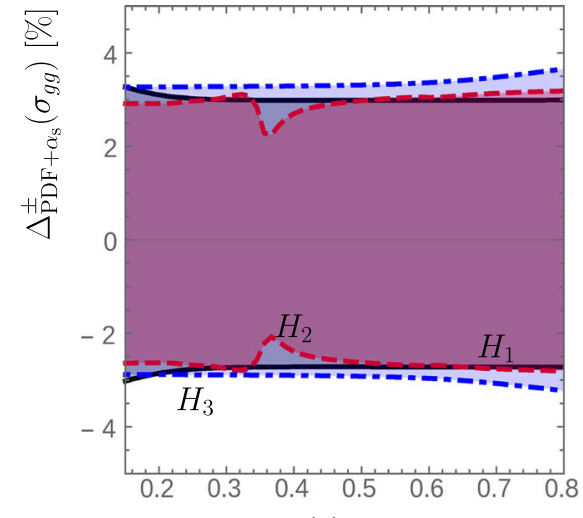

(a)

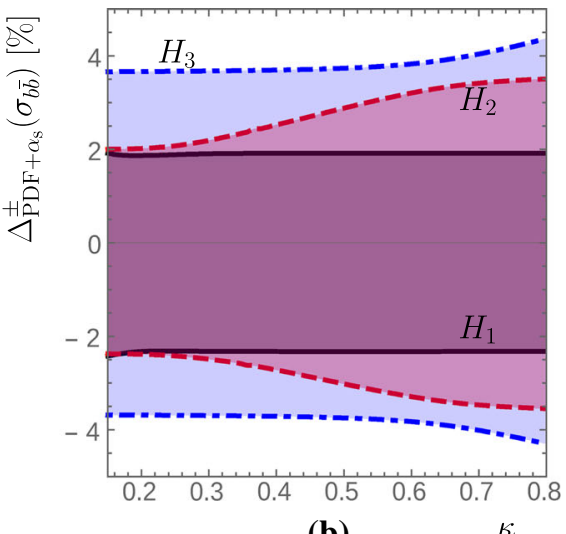

(b) top-quark effective theory. Electroweak corrections to gluon fusion mediated through light quarks are taken into account and the resummation of sbottom contributions for large values of $\tan \beta$ can be translated from the MSSM to the NMSSM. The latter procedure also applies to bottom-quark annihilation.

Our discussion comes along with an implementation of the neutral Higgs boson production cross section calculation in the code SusHi. The Higgs sector (obtained by an NMSSM spectrum generator) needs to be supplied through the SusHi input file. We briefly focused on the new features of the additional singlet-like CP-even or CP-odd Higgs boson for what concerns neutral Higgs boson production. Due to possible cancellations of quark-induced contributions, squark and electroweak corrections to gluon fusion can be of greater relevance than known in the MSSM, in particular for not too heavy third generation squark mass spectra. For a small singlet-doublet mixing term, which can be achieved by lowering the parameter $\lambda$, the singlet-like CP-even and -odd Higgs bosons can both be decoupled from the remaining MSSM-like Higgs sector. The renormalization and factorization scale uncertainties reflect the individual contributions to neutral Higgs boson production in the case of gluon fusion, whereas scale uncertainties for bottom-quark annihilation as well as PDF $+\alpha_{s}$ uncertainties for both production processes mainly remain a function of the Higgs boson mass.

We leave a more detailed investigation of theoretical uncertainties to future work. Moreover, interesting for future studies is an expansion in a light Higgs boson mass rather than heavy SUSY masses for what concerns the inclusion of NLO and NNLO SQCD contributions, in particular since for pure singlet-like CP-odd Higgs bosons NNLO stop-induced contributions are the first non-vanishing contributions to gluon fusion. Similarly a discussion of distributions and of the necessity of resummation for transverse momentum distributions is timely for the real NMSSM, but left for future work.
Acknowledgments The author thanks Jonathan Gaunt, Robert Harlander, Hendrik Mantler and Pietro Slavich for very helpful comments on the manuscript. The author is, moreover, indebted to Pietro Slavich for help in the translation of the MSSM NLO SQCD corrections to the NMSSM and to Robert Harlander and Hendrik Mantler for their comments on the implementation of the NMSSM in SusHi. The author also thanks Kathrin Walz for help related to the renormalization of the stop and sbottom sectors within NMSSMCALC and Peter Drechsel for help with regard to the calculation of NMSSM Higgs boson masses. The author acknowledges support by Deutsche Forschungsgemeinschaft (DFG) through the Collaborative Research Center SFB 676 "Particles, Strings and the Early Universe".

Open Access This article is distributed under the terms of the Creative Commons Attribution 4.0 International License (http://creativecomm ons.org/licenses/by/4.0/), which permits unrestricted use, distribution, and reproduction in any medium, provided you give appropriate credit to the original author(s) and the source, provide a link to the Creative Commons license, and indicate if changes were made.

Funded by $\mathrm{SCOAP}^{3}$.

\section{A Formulas: Higgs-squark-squark couplings in the NMSSM}

In this section we present the squark couplings to the five neutral Higgs bosons $\phi$ of the NMSSM as implemented in the code SusHi. As noted before, the singlet component $S$ does not couple to the quarks, such that the couplings of the Higgs bosons to the quarks can be taken over from the MSSM by replacing the mixing angle $\alpha$ and thus the projection on $H_{d}^{0}$ and $H_{u}^{0}$ by the proper mixing matrix elements $\mathcal{R}^{S} / \mathcal{R}^{P}$. On the contrary the singlet component couples to the squarks, for which we present the Feynman rules in the form

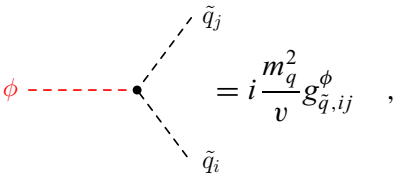

with $v=1 / \sqrt{\sqrt{2} G_{F}}=\sqrt{v_{d}^{2}+v_{u}^{2}}$. The couplings $g_{\tilde{q}, i j}^{\phi}$ of squarks with indices $\{i, j\}$ to CP-even Higgs bosons with 
$k=\{1,2,3\}$ or CP-odd Higgs bosons with $k=\{1,2\}$ are subsequently presented in the gauge eigenstates

$$
\begin{aligned}
g_{\tilde{q}, i j}^{H_{k}}= & \mathcal{R}_{k 1}^{S} \tilde{g}_{\tilde{q}, i j}^{H 1}+\mathcal{R}_{k 2}^{S} \tilde{g}_{\tilde{q}, i j}^{H 2}+\mathcal{R}_{k 3}^{S} \tilde{g}_{\tilde{q}, i j}^{H 3}, \\
g_{\tilde{q}, i j}^{A}= & \mathcal{R}_{k 1}^{P} \cdot\left(c_{\beta} \tilde{g}_{\tilde{q}, i j}^{A 1}-s_{\beta} \tilde{g}_{\tilde{q}, i j}^{A 2}\right) \\
& +\mathcal{R}_{k 2}^{P 2} \cdot\left(s_{\beta} \tilde{g}_{\tilde{q}, i j}^{A 1}+c_{\beta} \tilde{g}_{\tilde{q}, i j}^{A 2}\right)+\mathcal{R}_{k 3}^{P} \tilde{g}_{\tilde{q}, i j}^{A 3},
\end{aligned}
$$

where in the CP-odd sector the prerotation with $\mathcal{R}^{G}$ involving $c_{\beta}=\cos \beta$ and $s_{\beta}=\sin \beta$ is performed. They were obtained with the code MaCoR [111] and cross-checked against the formulas of Ref. [5] for what concerns the LO stop contributions to gluon fusion. The individual contributions $\tilde{g}_{\tilde{q}, i j}^{H k}$ in the CP-even sector with $k=\{1,2,3\}$ yield

$$
\begin{aligned}
& m_{b}^{2} \tilde{g}_{\tilde{b}, 11}^{H 1}=\frac{2 m_{b}^{2}}{c_{\beta}}-\frac{1}{6} m_{Z}^{2} c_{\beta}\left[3+c_{2 \theta_{b}}\left(1+2 c_{2 \theta_{W}}\right)\right] \\
& +\frac{s_{2 \theta_{b}}}{2 c_{\beta}}\left[\left(m_{\tilde{b}_{1}}^{2}-m_{\tilde{b}_{2}}^{2}\right) s_{2 \theta_{b}}+2 m_{b} \mu t_{\beta}\right], \\
& m_{b}^{2} \tilde{g}_{\tilde{b}, 11}^{H 2}=\frac{1}{6} m_{Z}^{2} s_{\beta}\left[3+c_{2 \theta_{b}}\left(1+2 c_{2 \theta_{W}}\right)\right]-\frac{s_{2 \theta_{b}}}{c_{\beta}} m_{b} \mu \text {, } \\
& m_{b} \tilde{g}_{\tilde{b}, 11}^{H 3}=-\frac{1}{\sqrt{2}} \lambda v s_{2 \theta_{b}} t_{\beta}, \\
& m_{b}^{2} \tilde{g}_{\tilde{b}, 12}^{H 1}=m_{b}^{2} \tilde{g}_{\tilde{b}, 21}^{H 1}=\frac{1}{6} m_{Z}^{2} s_{2 \theta_{b}} c_{\beta}\left(1+2 c_{2 \theta_{W}}\right) \\
& +\frac{c_{2 \theta_{b}}}{2 c_{\beta}}\left[\left(m_{\tilde{b}_{1}}^{2}-m_{\tilde{b}_{2}}^{2}\right) s_{2 \theta_{b}}+2 m_{b} \mu t_{\beta}\right], \\
& m_{b}^{2} \tilde{g}_{\tilde{b}, 12}^{H 2}=m_{b}^{2} \tilde{g}_{\tilde{b}, 21}^{H 2} \\
& =-\frac{1}{6} m_{Z}^{2} s_{2 \theta_{b}} s_{\beta}\left(1+2 c_{2 \theta_{W}}\right)-\frac{c_{2 \theta_{b}}}{c_{\beta}} m_{b} \mu, \\
& m_{b} \tilde{g}_{\tilde{b}, 12}^{H 3}=m_{b} \tilde{g}_{\tilde{b}, 21}^{H 3}=-\frac{1}{\sqrt{2}} \lambda v c_{2 \theta_{b}} t_{\beta}, \\
& m_{b}^{2} \tilde{g}_{\tilde{b}, 22}^{H 1}=\frac{2 m_{b}^{2}}{c_{\beta}}-\frac{1}{6} m_{Z}^{2} c_{\beta}\left[3-c_{2 \theta_{b}}\left(1+2 c_{2 \theta_{W}}\right)\right] \\
& -\frac{s_{2 \theta_{b}}}{2 c_{\beta}}\left[\left(m_{\tilde{b}_{1}}^{2}-m_{\tilde{b}_{2}}^{2}\right) s_{2 \theta_{b}}+2 m_{b} \mu t_{\beta}\right], \\
& m_{b}^{2} \tilde{g}_{\tilde{b}, 22}^{H 2}=\frac{1}{6} m_{Z}^{2} s_{\beta}\left[3-c_{2 \theta_{b}}\left(1+2 c_{2 \theta_{W}}\right)\right]+\frac{s_{2 \theta_{b}}}{c_{\beta}} m_{b} \mu, \\
& m_{b} \tilde{g}_{\tilde{b}, 22}^{H 3}=\frac{1}{\sqrt{2}} \lambda v s_{2 \theta_{b}} t_{\beta}, \\
& m_{t}^{2} \tilde{g}_{\tilde{t}, 11}^{H 1}=\frac{1}{6} m_{Z}^{2} c_{\beta}\left[3+c_{2 \theta_{t}}\left(-1+4 c_{2 \theta_{W}}\right)\right]-\frac{s_{2 \theta_{t}}}{s_{\beta}} m_{t} \mu, \\
& m_{t}^{2} \tilde{g}_{\tilde{t}, 11}^{H 2}=\frac{2 m_{t}^{2}}{s_{\beta}}-\frac{1}{6} m_{Z}^{2} s_{\beta}\left[3+c_{2 \theta_{t}}\left(-1+4 c_{2 \theta_{W}}\right)\right] \\
& +\frac{s_{2 \theta_{t}}}{2 s_{\beta}}\left[\left(m_{\tilde{t}_{1}}^{2}-m_{\tilde{t}_{2}}^{2}\right) s_{2 \theta_{t}}+2 m_{t} \mu \frac{1}{t_{\beta}}\right], \\
& m_{t} \tilde{g}_{\tilde{t}, 11}^{H 3}=-\frac{1}{\sqrt{2} t_{\beta}} \lambda v s_{2 \theta_{t}},
\end{aligned}
$$

$$
\begin{aligned}
m_{t}^{2} \tilde{g}_{\tilde{t}, 12}^{H 1}= & m_{t}^{2} \tilde{g}_{\tilde{t}, 21}^{H 1} \\
= & -\frac{1}{6} m_{Z}^{2} s_{2 \theta_{t}} c_{\beta}\left(-1+4 c_{2 \theta_{W}}\right)-\frac{c_{2 \theta_{t}}}{s_{\beta}} m_{t} \mu, \\
m_{t}^{2} \tilde{g}_{\tilde{t}, 12}^{H 2}= & m_{t}^{2} \tilde{g}_{\tilde{t}, 21}^{H 2}=\frac{1}{6} m_{t}^{2} s_{2 \theta_{t}} s_{\beta}\left(-1+4 c_{2 \theta_{W}}\right) \\
& +\frac{c_{2 \theta_{t}}}{2 s_{\beta}}\left[\left(m_{\tilde{t}_{1}}^{2}-m_{\tilde{t}_{2}}^{2}\right) s_{2 \theta_{t}}+2 m_{t} \mu \frac{1}{t_{\beta}}\right], \\
m_{t} \tilde{g}_{\tilde{t}, 12}^{H 3}= & m_{t} \tilde{g}_{\tilde{t}, 21}^{H 3}=-\frac{1}{\sqrt{2} t_{\beta}} \lambda v c_{2 \theta_{t}}, \\
m_{t}^{2} \tilde{g}_{\tilde{t}, 22}^{H 1}= & \frac{1}{6} m_{Z}^{2} c_{\beta}\left[3-c_{2 \theta_{t}}\left(-1+4 c_{2 \theta_{W}}\right)\right]+\frac{s_{2 \theta_{t}}}{s_{\beta}} m_{t} \mu, \\
m_{t}^{2} \tilde{g}_{\tilde{t}, 22}^{H 2}= & \frac{2 m_{t}^{2}}{s_{\beta}}-\frac{1}{6} m_{Z}^{2} s_{\beta}\left[3-c_{2 \theta_{t}}\left(-1+4 c_{2 \theta_{W}}\right)\right] \\
& -\frac{s_{2 \theta_{t}}}{2 s_{\beta}}\left[\left(m_{\tilde{t}_{1}}^{2}-m_{\tilde{t}_{2}}^{2}\right) s_{2 \theta_{t}}+2 m_{t} \mu \frac{1}{t_{\beta}}\right], \\
m_{t} \tilde{g}_{\tilde{t}, 22}^{H 3}= & \frac{1}{\sqrt{2} t_{\beta}} \lambda v s_{2 \theta_{t}} .
\end{aligned}
$$

In the CP-odd sector contributions with identical squark indices $\tilde{g}_{\tilde{q}, i i}^{A k}$ do not exist. The remaining ones are given by

$$
m_{b}^{2} \tilde{g}_{\tilde{b}, 12}^{A 1}=-m_{b}^{2} \tilde{g}_{\tilde{b}, 21}^{A 1}=\frac{1}{2 c_{\beta}}\left[\left(m_{\tilde{b}_{1}}^{2}-m_{\tilde{b}_{2}}^{2}\right) s_{2 \theta_{b}}+2 m_{b} \mu t_{\beta}\right],
$$$$
m_{b} \tilde{g}_{\tilde{b}, 12}^{A 2}=-m_{b} \tilde{g}_{\tilde{b}, 21}^{A 2}=\frac{\mu}{c_{\beta}},
$$$$
m_{b} \tilde{g}_{\tilde{b}, 12}^{A 3}=-m_{b} \tilde{g}_{\tilde{b}, 21}^{A 3}=\frac{1}{\sqrt{2}} \lambda v t_{\beta},
$$

$m_{t} \tilde{g}_{\tilde{t}, 12}^{A 1}=-m_{t} \tilde{g}_{\tilde{t}, 21}^{A 1}=\frac{\mu}{s_{\beta}}$,

$$
m_{t}^{2} \tilde{g}_{\tilde{t}, 12}^{A 2}=-m_{t}^{2} \tilde{g}_{\tilde{t}, 21}^{A 2}=\frac{1}{2 s_{\beta}}\left[\left(m_{\tilde{t}_{1}}^{2}-m_{\tilde{t}_{2}}^{2}\right) s_{2 \theta_{t}}+2 m_{t} \mu \frac{1}{t_{\beta}}\right],
$$

$m_{t} \tilde{g}_{\tilde{t}, 12}^{A 3}=-m_{t} \tilde{g}_{\tilde{t}, 21}^{A 3}=\frac{1}{\sqrt{2} t_{\beta}} \lambda v$.

All occurrences of the soft-breaking parameters $A_{t}$ and $A_{b}$ were replaced by their relation to the squark mixing angles $\theta_{b}$ and $\theta_{t}$. Trigonometric functions are abbreviated through $s_{x}=\sin x, c_{x}=\cos _{x}$, and $t_{x}=\tan x$.

\section{References}

1. G. Aad et al., (ATLAS Collaboration), Observation of a new particle in the search for the standard model Higgs boson with the ATLAS detector at the LHC. Phys. Lett. B 716, 1 (2012). arXiv: 1207.7214 
2. S. Chatrchyan et al., CMS Collaboration, Observation of a new boson at a mass of $125 \mathrm{GeV}$ with the CMS experiment at the LHC. Phys. Lett. B 716, 30 (2012). arXiv:1207.7235

3. U. Ellwanger, C. Hugonie, A.M. Teixeira, The next-to-minimal supersymmetric standard model. Phys. Rep. 496, 1 (2010). arXiv:0910.1785

4. M. Maniatis, The next-to-minimal supersymmetric extension of the standard model reviewed. Int. J. Mod. Phys. A 25, 3505 (2010). arXiv:0906.0777

5. S.F. King, M. Mühlleitner, R. Nevzorov, K. Walz, Natural NMSSM Higgs bosons. Nucl. Phys. B 870, 323 (2013). arXiv: 1211.5074

6. M. Spira, HIGLU: a program for the calculation of the total Higgs production cross-section at hadron colliders via gluon fusion including QCD corrections. arXiv:hep-ph/9510347

7. R.V. Harlander, S. Liebler, H. Mantler, SusHi: A program for the calculation of Higgs production in gluon fusion and bottom-quark annihilation in the standard model and the MSSM. Comput. Phys. Commun. 184, 1605 (2013). arXiv:1212.3249

8. S. Dittmaier et al., (LHC Higgs Cross Section Working Group Collaboration), Handbook of LHC Higgs Cross Sections: 1. Inclusive Observables. arXiv: 1101.0593

9. S. Dittmaier et al., (LHC Higgs Cross Section Working Group Collaboration), Handbook of LHC Higgs Cross Sections: 2. Differential Distributions. arXiv:1201.3084

10. S. Heinemeyer et al., (LHC Higgs Cross Section Working Group Collaboration), Handbook of LHC Higgs Cross Sections: 3. Higgs Properties. arXiv:1307.1347

11. H.M. Georgi, S.L. Glashow, M.E. Machacek, D.V. Nanopoulos, Higgs bosons from two gluon annihilation in proton proton collisions. Phys. Rev. Lett. 40, 692 (1978)

12. A. Djouadi, M. Spira, P.M. Zerwas, Production of Higgs bosons in proton colliders: QCD corrections. Phys. Lett. B 264, 440 (1991)

13. S. Dawson, Radiative corrections to Higgs boson production. Nucl. Phys. B 359, 283 (1991)

14. M. Spira, A. Djouadi, D. Graudenz, P.M. Zerwas, Higgs boson production at the LHC. Nucl. Phys. B 453, 17 (1995). arXiv:hep-ph/9504378

15. R.V. Harlander, W.B. Kilgore, Next-to-next-to-leading order Higgs production at hadron colliders. Phys. Rev. Lett. 88, 201801 (2002). arXiv:hep-ph/0201206

16. C. Anastasiou, K. Melnikov, Higgs boson production at hadron colliders in NNLO QCD. Nucl. Phys. B 646, 220 (2002). arXiv:hep-ph/0207004

17. V. Ravindran, J. Smith, W.L. van Neerven, NNLO corrections to the total cross-section for Higgs boson production in hadron hadron collisions. Nucl. Phys. B 665, 325 (2003). arXiv:hep-ph/0302135

18. S. Marzani, R.D. Ball, V. Del Duca, S. Forte, A. Vicini, Higgs production via gluon-gluon fusion with finite top mass beyond nextto-leading order. Nucl. Phys. B 800, 127 (2008). arXiv:0801.2544

19. R.V. Harlander, K.J. Ozeren, Finite top mass effects for hadronic Higgs production at next-to-next-to-leading order. JHEP 0911, 088 (2009). arXiv:0909.3420

20. A. Pak, M. Rogal, M. Steinhauser, Finite top quark mass effects in NNLO Higgs boson production at LHC. JHEP 1002, 025 (2010). arXiv:0911.4662

21. R.V. Harlander, H. Mantler, S. Marzani, K.J. Ozeren, Higgs production in gluon fusion at next-to-next-to-leading order QCD for finite top mass. Eur. Phys. J. C 66, 359 (2010). arXiv:0912.2104

22. A. Pak, M. Rogal, M. Steinhauser, Production of scalar and pseudo-scalar Higgs bosons to next-to-next-to-leading order at hadron colliders. JHEP 1109, 088 (2011). arXiv: 1107.3391

23. S. Catani, D. de Florian, M. Grazzini, P. Nason, Soft gluon resummation for Higgs boson production at hadron colliders. JHEP 0307, 028 (2003). arXiv:hep-ph/0306211
24. S. Moch, A. Vogt, Higher-order soft corrections to lepton pair and Higgs boson production. Phys. Lett. B 631, 48 (2005). arXiv:hep-ph/0508265

25. A. Idilbi, X. Ji, J. Ma, F. Yuan, Threshold resummation for Higgs production in effective field theory. Phys. Rev. D 73, 077501 (2006). arXiv:hep-ph/0509294

26. A. Idilbi, X. Ji, F. Yuan, Resummation of threshold logarithms in effective field theory for DIS, Drell-Yan and Higgs production. Nucl. Phys. B 753, 42 (2006). arXiv:hep-ph/0605068

27. V. Ravindran, Higher-order threshold effects to inclusive processes in QCD. Nucl. Phys. B 752, 173 (2006). arXiv:hep-ph/0603041

28. V. Ahrens, T. Becher, M. Neubert, L.L. Yang, Renormalizationgroup improved prediction for Higgs production at hadron colliders. Eur. Phys. J. C 62, 333 (2009). arXiv:0809.4283

29. S. Actis, G. Passarino, C. Sturm, S. Uccirati, NLO Electroweak corrections to Higgs boson production at hadron colliders. Phys. Lett. B 670, 12 (2008). arXiv:0809.1301

30. U. Aglietti, R. Bonciani, G. Degrassi, A. Vicini, Two loop light fermion contribution to Higgs production and decays. Phys. Lett. B 595, 432 (2004). arXiv:hep-ph/0404071

31. R. Bonciani, G. Degrassi, A. Vicini, On the generalized harmonic polylogarithms of one complex variable. Comput. Phys. Commun. 182, 1253 (2011). arXiv:1007.1891

32. R.D. Ball, M. Bonvini, S. Forte, S. Marzani, G. Ridolfi, Higgs production in gluon fusion beyond NNLO. Nucl. Phys. B 874, 746 (2013). arXiv: 1303.3590

33. D. de Florian, J. Mazzitelli, S. Moch, A. Vogt, Approximate $\mathrm{N}^{3}$ LO Higgs-boson production cross section using physical-kernel constraints. JHEP 1410, 176 (2014). arXiv:1408.6277

34. C. Anastasiou, C. Duhr, F. Dulat, E. Furlan, T. Gehrmann, F. Herzog, B. Mistlberger, Higgs boson gluonfusion production at threshold in $\mathrm{N}^{3}$ LO QCD. Phys. Lett. B 737, 325 (2014). arXiv: 1403.4616

35. C. Anastasiou, C. Duhr, F. Dulat, E. Furlan, T. Gehrmann, F. Herzog, B. Mistlberger, Higgs boson gluon-fusion production beyond threshold in $\mathrm{N}^{3}$ LO QCD. arXiv:1411.3584

36. G. Degrassi, P. Slavich, NLO QCD bottom corrections to Higgs boson production in the MSSM. JHEP 1011, 044 (2010). arXiv: 1007.3465

37. G. Degrassi, S. Di Vita, P. Slavich, NLO QCD corrections to pseudoscalar Higgs production in the MSSM. JHEP 1108, 128 (2011). arXiv:1107.0914

38. G. Degrassi, S. Di Vita, P. Slavich, On the NLO QCD corrections to the production of the heaviest neutral Higgs scalar in the MSSM. Eur. Phys. J. C 72, 2032 (2012). arXiv:1204.1016

39. R.V. Harlander, M. Steinhauser, Hadronic Higgs production and decay in supersymmetry at next-to-leading order. Phys. Lett. B 574, 258 (2003). arXiv:hep-ph/0307346

40. R.V. Harlander, M. Steinhauser, Supersymmetric Higgs production in gluon fusion at next-to-leading order. JHEP 0409, 066 (2004). arXiv:hep-ph/0409010

41. R.V. Harlander, F. Hofmann, Pseudo-scalar Higgs production at next-to-leading order SUSY-QCD. JHEP 0603, 050 (2006). arXiv:hep-ph/0507041

42. G. Degrassi, P. Slavich, On the NLO QCD corrections to Higgs production and decay in the MSSM. Nucl. Phys. B 805, 267 (2008). arXiv:0806.1495

43. R. Harlander, M. Steinhauser, Effects of SUSY QCD in hadronic Higgs production at next-to-next-to-leading order. Phys. Rev. D 68, 111701 (2003). arXiv:hep-ph/0308210

44. A. Pak, M. Steinhauser, N. Zerf, Towards Higgs boson production in gluon fusion to NNLO in the MSSM. Eur. Phys. J. C 71, 1602 (2011). arXiv:1012.0639 
45. A. Pak, M. Steinhauser, N. Zerf, Supersymmetric next-to-nextto-leading order corrections to Higgs boson production in gluon fusion. JHEP 1209, 118 (2012). arXiv: 1208.1588

46. E. Bagnaschi, R.V. Harlander, S. Liebler, H. Mantler, P. Slavich, A. Vicini, Towards precise predictions for Higgs-boson production in the MSSM. JHEP 1406, 167 (2014). arXiv:1404.0327

47. C. Anastasiou, S. Beerli, A. Daleo, The two-loop QCD amplitude $g g \rightarrow h, H$ in the minimal supersymmetric standard model. Phys. Rev. Lett. 100, 241806 (2008). arXiv:0803.3065

48. M. Mühlleitner, H. Rzehak, M. Spira, SUSY-QCD corrections to MSSM Higgs boson production via gluon fusion. PoS RADCOR 2009, 043 (2010). arXiv:1001.3214

49. C. Anastasiou, S. Beerli, S. Bucherer, A. Daleo, Z. Kunszt, Twoloop amplitudes and master integrals for the production of a Higgs boson via a massive quark and a scalar-quark loop. JHEP 0701, 082 (2007). arXiv:hep-ph/0611236

50. U. Aglietti, R. Bonciani, G. Degrassi, A. Vicini, Analytic results for virtual QCD corrections to Higgs production and decay. JHEP 0701, 021 (2007). arXiv:hep-ph/0611266

51. M. Mühlleitner, M. Spira, Higgs boson production via gluon fusion: squark loops at NLO QCD. Nucl. Phys. B 790, 1 (2008). arXiv:hep-ph/0612254

52. F. Maltoni, Z. Sullivan, S. Willenbrock, Higgs-boson production via bottom-quark fusion. Phys. Rev. D 67, 093005 (2003). arXiv:hep-ph/0301033

53. R.V. Harlander, W.B. Kilgore, Higgs boson production in bottom quark fusion at next-to-next-to leading order. Phys. Rev. D 68, 013001 (2003). arXiv:hep-ph/0304035

54. S. Dittmaier, M. Krämer, A. Mück, T. Schlüter, MSSM Higgsboson production in bottom-quark fusion: electroweak radiative corrections. JHEP 0703, 114 (2007). arXiv:hep-ph/0611353

55. S. Dawson, C.B. Jackson, P. Jaiswal, SUSY QCD corrections to Higgs-b production: is the $\Delta_{b}$ approximation accurate? Phys. Rev. D 83, 115007 (2011). arXiv:1104.1631

56. R.V. Harlander, H. Mantler, M. Wiesemann, Transverse momentum resummation for Higgs production via gluon fusion in the MSSM. JHEP 1411, 116 (2014). arXiv:1409.0531

57. K. Ender, T. Graf, M. Mühlleitner, H. Rzehak, Analysis of the NMSSM Higgs boson masses at one-loop level. Phys. Rev. D 85, 075024 (2012). arXiv: 1111.4952

58. A. Bartl, M. Hirsch, A. Vicente, S. Liebler, W. Porod, LHC phenomenology of the $\mu \nu$ SSM. JHEP 0905, 120 (2009). arXiv:0903.3596

59. M.S. Carena, D. Garcia, U. Nierste, C.E.M. Wagner, Effective Lagrangian for the $\bar{t} b H^{+}$interaction in the MSSM and charged Higgs phenomenology. Nucl. Phys. B 577, 88 (2000). arXiv:hep-ph/9912516

60. M.S. Carena, J.R. Ellis, S. Mrenna, A. Pilaftsis, C.E.M. Wagner, Collider probes of the MSSM Higgs sector with explicit CP violation. Nucl. Phys. B 659, 145 (2003). arXiv:hep-ph/0211467

61. J. Guasch, P. Häfliger, M. Spira, MSSM Higgs decays to bottom quark pairs revisited. Phys. Rev. D 68, 115001 (2003). arXiv:hep-ph/0305101

62. D. Noth, M. Spira, Higgs boson couplings to bottom quarks: two-loop supersymmetry-QCD corrections. Phys. Rev. Lett. 101, 181801 (2008). arXiv:0808.0087

63. D. Noth, M. Spira, Supersymmetric Higgs Yukawa couplings to bottom quarks at next-to-next-to-leading order. JHEP 1106, 084 (2011). arXiv:1001.1935

64. L. Mihaila, C. Reisser, $\mathcal{O}\left(\alpha_{s}^{2}\right)$ corrections to fermionic Higgs decays in the MSSM. JHEP 1008, 021 (2010). arXiv:1007.0693

65. J. Baglio, R. Gröber, M. Mühlleitner, D.T. Nhung, H. Rzehak, M. Spira, J. Streicher, K. Walz, NMSSMCALC: a program package for the calculation of loop-corrected Higgs boson masses and decay widths in the (complex) NMSSM. Comput. Phys. Commun. 185(12), 3372 (2014). arXiv: 1312.4788
66. R.V. Harlander, W.B. Kilgore, Production of a pseudoscalar Higgs boson at hadron colliders at next-to-next-to leading order. JHEP 0210, 017 (2002). arXiv:hep-ph/0208096

67. C. Anastasiou, R. Boughezal, F. Petriello, Mixed QCDelectroweak corrections to Higgs boson production in gluon fusion. JHEP 0904, 003 (2009). arXiv:0811.3458

68. E. Bagnaschi, G. Degrassi, P. Slavich, A. Vicini, Higgs production via gluon fusion in the POWHEG approach in the SM and in the MSSM. JHEP 1202, 088 (2012). arXiv: 1111.2854

69. P.Z. Skands, B.C. Allanach, H. Baer, C. Balazs, G. Belanger, F. Boudjema, A. Djouadi, R. Godbole et al., SUSY Les Houches accord: interfacing SUSY spectrum calculators, decay packages, and event generators. JHEP 0407, 036 (2004). arXiv:hep-ph/0311123

70. B.C. Allanach, C. Balazs, G. Belanger, M. Bernhardt, F. Boudjema, D. Choudhury, K. Desch, U. Ellwanger et al., SUSY Les Houches Accord 2. Comput. Phys. Commun. 180, 8 (2009). arXiv:0801.0045

71. U. Ellwanger, J.F. Gunion, C. Hugonie, NMHDECAY: a Fortran code for the Higgs masses, couplings and decay widths in the NMSSM. JHEP 0502, 066 (2005). arXiv:hep-ph/0406215

72. U. Ellwanger, C. Hugonie, NMHDECAY 2.0: an updated program for sparticle masses, Higgs masses, couplings and decay widths in the NMSSM. Comput. Phys. Commun. 175, 290 (2006). arXiv:hep-ph/0508022

73. G. Belanger, F. Boudjema, C. Hugonie, A. Pukhov, A. Semenov, Relic density of dark matter in the NMSSM. JCAP 0509, 001 (2005). arXiv:hep-ph/0505142

74. U. Ellwanger, C. Hugonie, NMSPEC: a Fortran code for the sparticle and Higgs masses in the NMSSM with GUT scale boundary conditions. Comput. Phys. Commun. 177, 399 (2007). arXiv:hep-ph/0612134

75. A. Djouadi, J. Kalinowski, M. Spira, HDECAY: a program for Higgs boson decays in the standard model and its supersymmetric extension. Comput. Phys. Commun. 108, 56 (1998). arXiv:hep-ph/9704448

76. T. Graf, R. Grober, M. Mühlleitner, H. Rzehak, K. Walz, Higgs boson masses in the complex NMSSM at one-loop level. JHEP 1210, 122 (2012). arXiv: 1206.6806

77. M. Mühlleitner, D.T. Nhung, H. Rzehak, K. Walz, Two-Loop Contributions of the Order $\mathcal{O}\left(\alpha_{\sqcup} \alpha_{f}\right)$ to the Masses of the Higgs Bosons in the CP-Violating NMSSM. arXiv: 1412.0918

78. B.C. Allanach, SOFTSUSY: a program for calculating supersymmetric spectra. Comput. Phys. Commun. 143, 305 (2002). arXiv:hep-ph/0104145

79. B.C. Allanach, P. Athron, L.C. Tunstall, A. Voigt, A.G. Williams, Next-to-minimal SOFTSUSY. Comput. Phys. Commun. 185, 2322 (2014). arXiv:1311.7659

80. F. Staub, SARAH 4: A tool for (not only SUSY) model builders. Comput. Phys. Commun. 185, 1773 (2014). arXiv:1309.7223

81. W. Porod, SPheno, a program for calculating supersymmetric spectra, SUSY particle decays and SUSY particle production at $e^{+} e^{-}$colliders. Comput. Phys. Commun. 153, 275 (2003). arXiv:hep-ph/0301101

82. W. Porod, F. Staub, SPheno 3.1: extensions including flavour, CPphases and models beyond the MSSM. Comput. Phys. Commun. 183, 2458 (2012). arXiv: 1104.1573

83. P. Athron, J. h. Park, D. Stöckinger and A. Voigt, FlexibleSUSYa spectrum generator generator for supersymmetric models. arXiv: 1406.2319

84. A. Brignole, G. Degrassi, P. Slavich, F. Zwirner, On the two loop sbottom corrections to the neutral Higgs boson masses in the MSSM. Nucl. Phys. B 643, 79 (2002). arXiv:hep-ph/0206101

85. S. Heinemeyer, W. Hollik, H. Rzehak, G. Weiglein, Highprecision predictions for the MSSM Higgs sector at $\mathcal{O}\left(\alpha_{b} \alpha_{s}\right)$. Eur. Phys. J. C 39, 465 (2005). arXiv:hep-ph/0411114 
86. S. Heinemeyer, H. Rzehak, C. Schappacher, Proposals for bottom quark/squark renormalization in the complex MSSM. Phys. Rev. D 82, 075010 (2010). arXiv:1007.0689

87. S. Schael et al., (ALEPH Collaboration, DELPHI Collaboration and L3 Collaboration), Search for neutral MSSM Higgs bosons at LEP. Eur. Phys. J. C 47, 547 (2006). arXiv:hep-ex/0602042

88. S. Chatrchyan et al., (CMS Collaboration), Search for a light pseudoscalar Higgs boson in the dimuon decay channel in $p p$ collisions at $\sqrt{s}=7$ TeV. Phys. Rev. Lett. 109, 121801 (2012). arXiv: 1206.6326

89. S. Chatrchyan et al., [CMS Collaboration], Search for a Higgs boson decaying into a b-quark pair and produced in association with b quarks in proton-proton collisions at $7 \mathrm{TeV}$. Phys. Lett. B 722, 207 (2013). arXiv:1302.2892

90. G. Aad et al., (ATLAS Collaboration), Search for a light charged Higgs boson in the decay channel $H^{+} \rightarrow c \bar{s}$ in $t \bar{t}$ events using pp collisions at $\sqrt{s}=7 \mathrm{STeV}$ with the ATLAS detector. Eur. Phys. J. C 73(6), 2465 (2013). arXiv: 1302.3694

91. G. Aad et al., ATLAS Collaboration, Search for top quark decays $t \rightarrow q H$ with $H \rightarrow \gamma \gamma$ using the ATLAS detector. JHEP 1406, 008 (2014). arXiv:1403.6293

92. S. Chatrchyan et al., (CMS Collaboration), Search for invisible decays of Higgs bosons in the vector boson fusion and associated $Z H$ production modes. Eur. Phys. J. C 74, 2980 (2014). arXiv: 1404.1344

93. G. Aad et al., ATLAS Collaboration, Search for scalar diphoton resonances in the mass range $65-600 \mathrm{GeV}$ with the ATLAS detector in $p p$ collision data at $\sqrt{s}=8 \mathrm{TeV}$. Phys. Rev. Lett. 113(17), 171801 (2014). arXiv:1407.6583

94. V. Khachatryan et al., (CMS Collaboration), Search for neutral MSSM Higgs bosons decaying to a pair of tau leptons in pp collisions. JHEP 1410, 160 (2014). arXiv: 1408.3316

95. G. Aad et al., ATLAS Collaboration, Search for neutral Higgs bosons of the minimal supersymmetric standard model in pp collisions at $\sqrt{s}=8 \mathrm{TeV}$ with the ATLAS detector. JHEP 1411, 056 (2014). arXiv:1409.6064

96. V. Khachatryan et al., CMS Collaboration, Searches for heavy Higgs bosons in two-Higgs-doublet models and for $t \rightarrow c h$ decay using multilepton and diphoton final states in $p p$ collisions at 8 TeV. Phys. Rev. D 90(11), 112013 (2014). arXiv:1410.2751

97. G. Aad et al., ATLAS Collaboration, Search for a CP-odd Higgs boson decaying to $Z h$ in $p p$ collisions at $\sqrt{s}=8 \mathrm{TeV}$ with the ATLAS detector. arXiv: 1502.04478
98. CMS Collaboration (CMS Collaboration), Search for an Higgs like resonance in the diphoton mass spectra above $150 \mathrm{GeV}$ with $8 \mathrm{TeV}$ data. CMS-PAS-HIG-14-006

99. CMS Collaboration (CMS Collaboration), Search for a pseudoscalar boson $A$ decaying into a $Z$ and an $h$ boson in the llbb final state. CMS-PAS-HIG-14-011

100. G. Aad et al., (ATLAS Collaboration), Search for invisible decays of a Higgs boson produced in association with a $Z$ boson in ATLAS. Phys. Rev. Lett. 112, 201802 (2014). arXiv:1402.3244

101. The ATLAS collaboration, Search for charged Higgs bosons decaying via $H^{ \pm} \rightarrow \tau^{ \pm} v$ in hadronic final states using $p p$ collision data at $\sqrt{s}=8 \mathrm{TeV}$ with the ATLAS detector. ATLASCONF-2014-050, ATLAS-COM-CONF-2014-071

102. CMS Collaboration (CMS Collaboration), Search for charged Higgs bosons with the $\mathrm{H}+$ to tau nu decay channel in the fully hadronic final state at $\sqrt{s}=8 \mathrm{TeV}$. CMS-PAS-HIG-14-020

103. A.D. Martin, W.J. Stirling, R.S. Thorne, G. Watt, Parton distributions for the LHC. Eur. Phys. J. C 63, 189 (2009). arXiv:0901.0002

104. S. Dittmaier, M. Krämer, M. Spira, Higgs radiation off bottom quarks at the Tevatron and the CERN LHC. Phys. Rev. D 70, 074010 (2004). arXiv:hep-ph/0309204

105. S. Dawson, C.B. Jackson, L. Reina, D. Wackeroth, Exclusive Higgs boson production with bottom quarks at hadron colliders. Phys. Rev. D 69, 074027 (2004). arXiv:hep-ph/0311067

106. S. Alekhin, S. Alioli, R.D. Ball, V. Bertone, J. Blümlein, M. Botje, J. Butterworth, F. Cerutti et al., The PDF4LHC Working Group Interim Report. arXiv:1101.0536

107. M. Botje, J. Butterworth, A. Cooper-Sarkar, A. de Roeck, J. Feltesse, S. Forte, A. Glazov, J. Huston et al., The PDF4LHC Working Group Interim Recommendations. arXiv:1101.0538

108. L. A. Harland-Lang, A.D. Martin, P. Motylinski, R.S. Thorne, Parton distributions in the LHC era: MMHT 2014 PDFs. arXiv: 1412.3989

109. R.D. Ball et al., (The NNPDF Collaboration), Parton distributions for the LHC Run II. arXiv: 1410.8849

110. H.L. Lai, M. Guzzi, J. Huston, Z. Li, P.M. Nadolsky, J. Pumplin, C.-P. Yuan, New parton distributions for collider physics. Phys. Rev. D 82, 074024 (2010). arXiv: 1007.2241

111. S. Liebler, W. Porod, Electroweak corrections to neutralino and chargino decays into a W-boson in the (N)MSSM. Nucl. Phys. B 849, 213 (2011). arXiv:1011.6163 (erratum-ibid. B 856, 125, 2012) 